Domenico Mucci

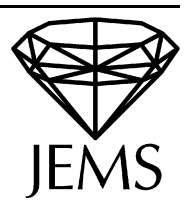

\title{
A characterization of graphs which can be approximated in area by smooth graphs
}

Received April 29, 1999 / final version received July 21, 2000

Published online September 25, 2000 - (c) Springer-Verlag \& EMS 2000

Abstract. For vector valued maps, convergence in $W^{1,1}$ and of all minors of the Jacobian matrix in $L^{1}$ is equivalent to convergence weakly in the sense of currents and in area for graphs. We show that maps defined on domains of dimension $n \geq 3$ can be approximated strongly in this sense by smooth maps if and only if the same property holds for the restriction to a.e. 2-dimensional plane intersecting the domain.

This paper deals with strong approximability of vector valued maps and jointly with the relaxed extension of the nonparametric area functional.

Let $\Omega$ be a bounded domain in $\mathbf{R}^{n}, n, N \geq 2$, and $u: \Omega \rightarrow \mathbf{R}^{N}$ be a smooth map. The area of the graph of $u$ over $\Omega$ is given by

$$
\mathrm{A}(u, \Omega):=\int_{\Omega}|M(D u)| d x
$$

where

$$
|M(D u)|:=\left(1+|D u|^{2}+\left|M_{(2)}(D u)\right|^{2}+\cdots+\left|M_{(\underline{n})}(D u)\right|^{2}\right)^{1 / 2}
$$

is the square root of the sum of the squares of the determinants of all minors of the Jacobian matrix $D u$ up to the order $\underline{n}:=\min (n, N)$. More precisely, if $I d \bowtie u: \Omega \rightarrow \Omega \times \mathbf{R}^{N}$ is the join map $(I \bar{d} \bowtie u)(x):=(x, u(x)),|M(D u)|$ is the $n$-dimensional Jacobian of $I d \bowtie u$ or, equivalently, the norm of the $n$-vector orienting the tangent $n$-space to the graph at $(x, u(x))$

$$
M(D u):=\left(e_{1}+\sum_{j=1}^{N} D_{1} u^{j} \varepsilon_{j}\right) \wedge \ldots \wedge\left(e_{n}+\sum_{j=1}^{N} D_{n} u^{j} \varepsilon_{j}\right)
$$

$\left\{e_{i}\right\}_{i=1}^{n},\left\{\varepsilon_{j}\right\}_{i=1}^{N}$ being canonical basis for $\mathbf{R}^{n}$ and $\mathbf{R}^{N}$, respectively.

D. Mucci: Dipartimento di Matematica, Università di Parma, Via M. D’Azeglio 85/A, 43100 Parma, Italy; e-mail: mucci@ prmat.math.unipr.it

Mathematics Subject Classification (1991): 49Q20, 49Q15 
In the sequel we will say that a sequence of smooth maps $\left\{u_{k}\right\}$ converges strongly in $L^{1}$ with all minors to $u$, if $u_{k}$ converges to $u$ in $W^{1,1}$ and all minors of the Jacobian matrix $D u_{k}$ converge in $L^{1}$ to the corresponding minors of $D u$, i.e.,

$$
\begin{cases}u_{k} \rightarrow u & \text { strongly in } L^{1} \\ M\left(D u_{k}\right) \rightarrow M(D u) & \text { strongly in } L^{1}\end{cases}
$$

We consider the problem of defining the class of non regular maps which can be approximated by smooth maps strongly in $L^{1}$ with all minors.

Of course, any approximable map belongs to the class $\mathcal{A}^{1}\left(\Omega, \mathbf{R}^{N}\right)$ of maps $u \in W^{1,1}\left(\Omega, \mathbf{R}^{N}\right)$ such that all minors of $D u$ are summable in $\Omega$, consequently we can still define $\mathrm{A}(u, \Omega)$ by (1).

For $u \in \mathcal{A}^{1}\left(\Omega, \mathbf{R}^{N}\right)$, it can be well defined the integer multiplicity (i.m.) rectifiable current $G_{u} \in \mathcal{R}_{n}\left(\Omega \times \mathbf{R}^{N}\right)$ "carried" by the graph of $u$, see [3], [7] and [8, Vol. I, Ch. 3]. More precisely, taking a.e. approximate derivatives we have

$$
G_{u}:=(I d \bowtie u)_{\#} \llbracket \Omega \rrbracket
$$

i.e., if $\omega \in \mathcal{D}^{n}\left(\Omega \times \mathbf{R}^{N}\right)$ is any smooth $n$-form with compact support in $\Omega \times \mathbf{R}^{N}$, $G_{u}$ acts on $\omega$ by

$$
G_{u}(\omega)=\int_{\Omega}(I d \bowtie u)^{\#} \omega d x=\int_{\Omega}<\omega(x, u(x)), M(D u(x))>d x
$$

where $<\cdot, \cdot>$ is the duality between $n$-covectors and $n$-vectors in $\mathbf{R}^{n} \times \mathbf{R}^{N}$. Therefore, the mass of $G_{u}$ is equal to the area of the graph of $u$

$$
\mathbf{M}\left(G_{u}\right)=\mathrm{A}(u, \Omega) .
$$

Trivially, strong approximation (2) implies approximation of graphs weakly in the sense of currents

$$
G_{u_{k}} \rightarrow G_{u}
$$

i.e., in dual sense

$$
\lim _{k \rightarrow+\infty} G_{u_{k}}(\omega)=G_{u}(\omega) \quad \forall \omega \in \mathcal{D}^{n}\left(\Omega \times \mathbf{R}^{N}\right) .
$$

Moreover, (2) improves the standard lower semicontinuity of mass w.r.t. weak convergence (3) to mass convergence

$$
\lim _{k \rightarrow+\infty} \mathbf{M}\left(G_{u_{k}}\right)=\mathbf{M}\left(G_{u}\right) .
$$

Also, for smooth maps, Stokes theorem implies that $G_{u}$ has no boundary in $\Omega \times \mathbf{R}^{N}$

$$
\partial G_{u}\left\llcorner\Omega \times \mathbf{R}^{N}=0\right.
$$


condition which is clearly preserved by weak convergence (3). Since in general for maps $u \in \mathcal{A}^{1}\left(\Omega, \mathbf{R}^{N}\right)$ (5) does not hold, compare [4] and [5], we are therefore led to seek for approximable maps in the special class of Cartesian maps

$$
\operatorname{cart}^{1}\left(\Omega, \mathbf{R}^{N}\right):=\left\{u \in W^{1,1}\left(\Omega, \mathbf{R}^{N}\right) \mid M(D u) \in L^{1}, \partial G_{u}\left\llcorner\Omega \times \mathbf{R}^{N}=0\right\} .\right.
$$

On the other hand, as noticed in [1], a theorem due to Reshetnyak yields that the apparently weaker approximation in area for graphs, i.e., (3) and (4) together, implies, and therefore is equivalent to, strong approximation (2). In fact (3) and (4) yield convergence weakly in $B V$-sense of $u_{k}$ to $u$ and weakly as measures of all minors of $D u_{k}$ to the minors of $D u$, thus it suffices to apply the continuity theorem in [15], see [1, Thm. 2.2].

These facts lead us to introduce the notion of relaxed area of the graph of a map $u \in \operatorname{cart}^{1}\left(\Omega, \mathbf{R}^{N}\right)$ w.r.t. weak convergence as currents

$$
\widetilde{\mathrm{A}}(u, \Omega):=\inf \left\{\liminf _{k \rightarrow+\infty} \mathrm{A}\left(u_{k}, \Omega\right) \mid u_{k} \in C^{1}\left(\Omega, \mathbf{R}^{N}\right) \text { and } G_{u_{k}} \rightarrow G_{u}\right\} .
$$

By lower semicontinuity of mass one has

$$
\mathrm{A}(u, \Omega) \leq \widetilde{\mathrm{A}}(u, \Omega)
$$

and therefore equality in (7) holds if and only if $u$ can be approximated strongly in $L^{1}$ with all minors by smooth maps.

In spite of that, it is shown in [6], see also [11], that in general

$$
\mathrm{A}(u, \Omega)<\widetilde{\mathrm{A}}(u, \Omega) .
$$

More precisely, if $B^{n}$ is the unit ball in $\mathbf{R}^{n}$, for $n=2$ there exist maps $u \in$ $\operatorname{cart}^{1}\left(B^{2}, \mathbf{R}^{2}\right)$ which are smooth, $C^{\infty}$, outside the origin, such that for any sequence of smooth maps $u_{k}: B^{2} \rightarrow \mathbf{R}^{2}$, with graphs $G_{u_{k}}$ weakly converging to $G_{u}$, one has

$$
\liminf _{k \rightarrow+\infty} \mathbf{M}\left(G_{u_{k}}\right)>\mathbf{M}\left(G_{u}\right)+\varepsilon
$$

where $\varepsilon$ is a positive constant independent of $\left\{u_{k}\right\}$.

Lack of approximability, in these cases, depends on the nontrivial homotopy type of loops $u_{\mid \partial B_{\rho}^{2}}$, where $B_{\rho}^{n}$ is the ball of radius $\rho$ and $\partial B_{\rho}^{n}$ its boundary. For example, taking the homogeneous extension

$$
u(x):=\varphi\left(\frac{x}{|x|}\right), \quad x \in B^{2} \backslash\{0\},
$$

where $\varphi: S^{1} \rightarrow \mathbf{R}^{2}, S^{1}=\partial B^{2}$, is the map defined in terms of the angle $\theta$ by

$$
\varphi(\theta):=\left\{\begin{array}{lll}
(-1+\cos 4 \theta, \sin 4 \theta) & \text { if } & 0 \leq \theta<\pi / 2 \\
(1-\cos 4 \theta, \sin 4 \theta) & \text { if } & \pi / 2 \leq \theta<\pi \\
(-1+\cos 4 \theta,-\sin 4 \theta) & \text { if } & \pi \leq \theta<3 \pi / 2 \\
(1-\cos 4 \theta,-\sin 4 \theta) & \text { if } & 3 \pi / 2 \leq \theta<2 \pi
\end{array}\right.
$$


clearly $u \in W^{1, p}$ for $p<2$ and $\operatorname{det} D u=0$ in $B^{2}$, hence $u \in \mathcal{A}^{1}\left(B^{2}, \mathbf{R}^{2}\right)$. Moreover

$$
\partial G_{u}\left\llcorner B^{2} \times \mathbf{R}^{2}=-\delta_{0} \times \varphi_{\#} \llbracket S^{1} \rrbracket\right.
$$

where $\delta_{x}$ denotes the Dirac mass centered at $x$, so that since the image of $S^{1}$ through $\varphi$ is the boundary of the union of the two unit disks of the target space $\mathbf{R}^{2}$ centered in $(-1,0)$ and $(1,0)$, and $\varphi\left(S^{1}\right)$ is covered twice with opposite orientation, one has $\varphi_{\#} \llbracket\left[S^{1} \rrbracket=0\right.$ and then $u \in \operatorname{cart}^{1}\left(B^{2}, \mathbf{R}^{2}\right)$. Notwithstanding, $\varphi$ is not homotopic to a constant in the domain

$$
\widehat{\Omega}=B((0,0), 2) \backslash[\bar{B}((1,0), 3 / 4) \cup \bar{B}((-1,0), 3 / 4)]
$$

whereas strong $L^{1}$-convergence with all minors (2) preserves geometric properties such as null-homotopy of smooth graphs, compare Theorem 1 in Sect. 3.4.2 of [8, Vol. I]. Therefore, if $u$ were approximable by smooth maps, $u_{\mid \partial B_{\rho}^{2}}$ would be homotopic in $\widehat{\Omega}$ to a constant map for a.e. $0<\rho<1$, which gives a contradiction.

Dealing with counterexamples to approximation, in [14] it is shown that there exist Cartesian maps $u$ such that for any sequence $\left\{u_{k}\right\}$ of smooth maps with graphs $G_{u_{k}}$ weakly converging to $G_{u}$, then the masses cannot be equibounded, i.e., $\mathbf{M}\left(G_{u_{k}}\right) \rightarrow+\infty$ as $k \rightarrow+\infty$.

It remains an open question to characterize approximable maps in case of dimension $n=2$. However, geometric obstructions are essentially due to the first homotopy group, see [9].

In fact, given a Lipschitz map $f: \partial B^{n} \rightarrow \mathbf{R}^{N}$ and an i.m. polyhedral $n$ dimensional chain $T$ such that $\partial T=f_{\#} \llbracket \partial B^{n} \rrbracket$, the previous example (with $T=0$ ) shows that if $n=2$, in general it cannot be found a Lipschitz extension of $f$ to $\bar{B}^{n}$ with mapping area comparable to the mass of $T$.

On the other hand, in case $n \geq 3$, taking account of Hurewitz Isomorphism theorem [9], B. White showed in [17] that, with the previous hypotheses, there exists a Lipschitz map $F: \bar{B}^{n} \rightarrow \mathbf{R}^{N}$, with $F_{\mid \partial B^{n}}=f$, such that each $n$-simplex that occurs with multiplicity $k$ in $T$ is covered $\leq k$ times (with the proper orientation) by $F$ and

$$
\operatorname{area}(F) \leq \mathbf{M}(T) .
$$

An adaptation of [17, Prop. 1 and Thm. 1] to our context, compare Proposition 1 in Sect. 1, suggests that roughly speaking obstructions to approximation are due to the nontriviality of 1-dimensional loops which "lie" on the graph, and therefore can be read on the 2-dimensional graphs given by sectioning the domain with 2-planes.

In this paper we show that this is actually the case. More precisely, let $E_{2}$ be any coordinate 2-dimensional plane of $\mathbf{R}^{n}$ and, for $x \in\left(E_{2}\right)^{\perp}$, let $\Pi_{x}$ denote the affine plane parallel to $E_{2}$ and containing $x, \Pi_{x}:=x+E_{2}$. Also, in case $\Omega_{x}:=\Pi_{x} \cap \Omega \neq \emptyset$, denote by $u_{\mid \Omega_{x}}: \Omega_{x} \rightarrow \mathbf{R}^{N}$ the restriction of $u$ to $\Omega_{x}$. Finally, $\mathcal{H}^{k}$ is the $k$-dimensional Hausdorff measure in $\mathbf{R}^{n}$.

We are going to prove the following

Main result. Let $u: \Omega \rightarrow \mathbf{R}^{N}$ be a vector valued map defined on a bounded domain $\Omega \subset \mathbf{R}^{n}$, with $n \geq 3$ and $N \geq 2$. Then $u$ can be approximated strongly in $L^{1}$ with all minors by smooth maps, i.e., there exists a sequence $\left\{u_{k}\right\}$ of smooth 
maps $u_{k}: \Omega \rightarrow \mathbf{R}^{N}$ for which (2) holds, if and only if $u$ is in $\operatorname{cart}^{1}\left(\Omega, \mathbf{R}^{N}\right)$ and for any coordinate plane $E_{2}$ of $\mathbf{R}^{n}$, for $\mathcal{H}^{n-2}$-a.e. $x \in\left(E_{2}\right)^{\perp}$, the restriction $u_{\mid \Omega_{x}}$ can be approximated by smooth maps.

The "only if" part is trivial since, as previously shown, if $u$ is approximable then $u \in \operatorname{cart}^{1}\left(\Omega, \mathbf{R}^{N}\right)$ and, by Fubini's theorem, for a.e. choiche of $\Omega_{x}, u_{\mid \Omega_{x}}$ is in $\operatorname{cart}^{1}\left(\Omega_{x}, \mathbf{R}^{N}\right)$ and is approximated by smooth maps $\left\{u_{k \mid \Omega_{x}}\right\}$. Note that, in terms of currents, we are saying that Cartesian graphs can be approximated in area by smooth graphs, i.e., equality holds in (7), if the same holds for a.e. 2-dimensional graph obtained by slicing the domain.

Since by [13] each continuous Cartesian map can be approximated by smooth maps, we easily infer the following extension of [12]

Corollary 1. If $u \in \operatorname{cart}^{1}\left(\Omega, \mathbf{R}^{N}\right), \Omega \subset \mathbf{R}^{n}, n \geq 3$, and the set of discontinuity points of $u$ has Hausdorf dimension strictly smaller than $n-2$, then $u$ can be approximated strongly in $L^{1}$ with all minors by smooth maps.

The rest of the paper divides in five sections. In Sect. 1 we extend the definition of Cartesian maps to polyhedral regions, outlining the main features, and prove an extension of White's results [17] to the setting of Cartesian maps, see Proposition 1. In Sect. 2 we study some local properties of approximable 2-dimensional graphs, see Proposition 2. In Sect. 3, taking account of Sect. 2, we give the geometric description of the local approximation argument, see Proposition 3. In Sect. 4 we describe a suitable partition of the "singular" open set $U \subset \Omega$ of a map $u$ in $\operatorname{cart}^{1}\left(\Omega, \mathbf{R}^{N}\right)$, thus obtaining nice integral estimates for each polyhedral region of the partition, see Proposition 4. To this aim we make use of the notion of Whitney family of cubes, see [2]. In Sect. 5, to prove the "if" part, we first define the singular set $U$ of $u$, then partition $U$ according to Sect. 4 and finally make an adaptation of the local approximation argument of Sect. 3.

\section{Definitions and preliminary results}

In the sequel, $Q$ will denote a $k$-dimensional polyhedral region of $\mathbf{R}^{n}$ (non convex, in general) with sides parallel to the coordinate $(k-1)$-planes and which is contained in a $k$-dimensional subspace of $\mathbf{R}^{n}$. For example, in Sect. 3 we take $Q:=[-\delta, \delta]^{n}$, with $0<\delta<1$. Also, let $Q_{(j)}$ denote the $j$-dimensional skeleton of $Q$, i.e., the set of $j$-faces of $Q, j=0, \ldots, k$. For the sake of simplicity, in the sequel $Q_{(j)}$ will also denote the set of points of the $j$-skeleton, thus $Q_{(k-1)}=\partial Q$.

We first extend in a natural way the notion of Cartesian map to polyhedral regions, outlining the main properties.

Definition 1. Let $w: U \subset \mathbf{R}^{n} \rightarrow \mathbf{R}^{N}$ be a map in $W^{1,1}\left(U, \mathbf{R}^{N}\right)$, where $U$ is either a polyhedral region $Q$ or its boundary $\partial Q$ or $U=[0,1] \times \partial Q$. Moreover, suppose that all minors of the Jacobian matrix of $D w$ are in $L^{1}$, where $D w$ is the differential of $w$ w.r.t. an orthonormal basis for $U$. Finally, let

$$
G_{w}=G_{w}\left\llcorner U \times \mathbf{R}^{N}=(I d \bowtie w)_{\#} \llbracket U \rrbracket\right.
$$


denote the i.m. rectifiable current in $\mathcal{R}_{k}\left(\mathbf{R}^{n} \times \mathbf{R}^{N}\right)$, with $k=\operatorname{dim}(U)$, carried by the graph $I d \bowtie w(U)$. More precisely, if $|M(D w)|$ denotes the a.e. defined $k$-dimensional Jacobian of the join map $I d \bowtie w: U \rightarrow U \times \mathbf{R}^{N}$

$$
|M(D w)|:=\left(1+|D w|^{2}+\left|M_{(2)}(D w)\right|^{2}+\cdots+\left|M_{(\underline{n})}(D w)\right|^{2}\right)^{1 / 2}
$$

where $\left|M_{(j)}(D w)\right|^{2}$ is the sum of the squares of the determinants of the minors of order $j$ of the Jacobian matrix of $D w$, for $j \leq \underline{n}:=\min (k, N)$, we have

$$
\mathbf{M}\left(G_{w}\left\llcorner U \times \mathbf{R}^{N}\right)=\int_{U}|M(D w)| d \mathcal{H}^{k} .\right.
$$

We say that $w$ is a Cartesian map in $\operatorname{cart}^{1}\left(U, \mathbf{R}^{N}\right)$, if one also has

$$
\partial G_{w}\left\llcorner\operatorname{int}(U) \times \mathbf{R}^{N}=0\right.
$$

in case $\partial U \neq \emptyset$, where $\operatorname{int}(U)=\operatorname{clos}(U) \backslash \partial U$, and $\partial G_{w}=0$ in case $U$ is a boundary, i.e., $\partial U=0$. In particular, if in addition the restriction $w_{\mid \partial U}$ of $w$ to the boundary of $U$ is a map in $\operatorname{cart}^{1}\left(\partial U, \mathbf{R}^{N}\right)$, one has

$$
\begin{aligned}
& \partial\left(G_{w}\left\llcorner U \times \mathbf{R}^{N}\right)=G_{w_{\mid \partial U}}\left\llcorner\partial U \times \mathbf{R}^{N}\right.\right. \\
& \quad=(I d \bowtie w)_{\mid \partial U \# \rrbracket} \llbracket \partial U \rrbracket \in \mathcal{R}_{k-1}\left(\mathbf{R}^{n} \times \mathbf{R}^{N}\right)
\end{aligned}
$$

and

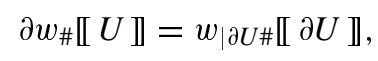

compare 3.2.5 and 3.2.3 of [8, Vol. I].

Let now $u$ be a map in $\operatorname{cart}^{1}\left(\Omega, \mathbf{R}^{N}\right), \Omega \subset \mathbf{R}^{n}$ a bounded domain, and $Q$ be a $k$-dimensional polyhedral region strictly contained in $\Omega, Q \subset \subset \Omega$.

Definition 2. We say that $Q$ is in generic position w.r.t. the map $u$ if for each $(k-j)$-face $K$ of $Q_{(k-j)}, j=1, \ldots, k-1$, the restriction of $u$ to $K, u_{K}:=u_{\mid K}$ : $K \rightarrow \mathbf{R}^{N}$, is a map in $\operatorname{cart}^{1}\left(K, \mathbf{R}^{N}\right)$. In this case, by Definition 1 , for any such $K$ we have $\partial G_{u_{K}} L \operatorname{int}(K) \times \mathbf{R}^{N}=0$ and

$$
\int_{K}\left|M\left(D u_{K}\right)\right| d \mathcal{H}^{k-j}<+\infty
$$

Remark 1. Note that by slight movements of the faces of $Q$, which do not affect the size, we can in general require $Q$ to be as in Definition 2 .

As cited in the introduction, to prove Proposition 3 in Sect. 3, we make use of the following generalization of [17, Thm. 1] 
Proposition 1. Let $v: \Omega \rightarrow \mathbf{R}^{N}$ be a continuous map in $\operatorname{cart}^{1}\left(\Omega, \mathbf{R}^{N}\right), \Omega \subset \mathbf{R}^{n}$, $n \geq 3$. Denote by $Q$ the $k$-cube $Q:=[-1,1]^{k} \times\left\{0_{\mathbf{R}^{n-k}}\right\}$, where $k \geq 3$. Also, suppose $Q$ is strictly contained in $\Omega$ and in generic position w.r.t. $v$ (see Definitions 1 and 2). Let $T$ be an i.m. rectifiable $k$-current in $\mathbf{R}^{n+N}, T \in \mathcal{R}_{k}\left(\mathbf{R}^{n+N}\right)$, such that

$$
\partial T=G_{v \mid \partial Q}\left\llcorner\partial Q \times \mathbf{R}^{N}=(I d \bowtie v)_{\mid \partial Q \# \llbracket \partial Q \rrbracket .}\right.
$$

Then, for each small $\varepsilon>0$, there exists a map $w: Q \rightarrow \mathbf{R}^{N}$, continuous up to the closure of $Q$, in $\operatorname{cart}^{1}\left(Q, \mathbf{R}^{N}\right)$, satisfying

$$
\partial\left(G_{w}\left\llcorner Q \times \mathbf{R}^{N}\right)=G_{v \mid \partial Q}\left\llcorner\partial Q \times \mathbf{R}^{N}\right.\right.
$$

(hence $w_{\mid \partial Q}=v$ ), such that its mapping area is comparable to the mass of $T$, i.e.,

$$
\int_{Q}\left|M_{(k)}(D w)\right| d x \leq \mathbf{M}(T)+\varepsilon,
$$

provided $N \geq k$, where $\left|M_{(k)}(D w)\right|$ is the $k$-dimensional Jacobian of $w$ (coinciding with $\left|\operatorname{det} D w_{\mid Q}\right|$ if $N=k$ ) whereas, if $N<k,\left|M_{(k)}(D w)\right| \equiv 0$, thus the $k$-dimensional mapping area of $w$ is zero.

Proof. If $f: \partial Q \rightarrow \mathbf{R}^{n+N}$ is a Lipschitz map and $\widetilde{T} \in \mathcal{R}_{k}\left(\mathbf{R}^{n+N}\right)$ is an i.m. rectifiable current with $\partial \widetilde{T}=f_{\#} \llbracket \partial Q \rrbracket$, by [17, Thm. 1 and Prop. 1], there exists a Lipschitz map $g: Q \rightarrow \mathbf{R}^{n+N}$ such that $g_{\mid \partial Q}=f$ and with $k$-dimensional mapping area comparable to the mass of $\widetilde{T}$, i.e.,

$$
\int_{Q} J_{k} g d x \leq \mathbf{M}(\widetilde{T})+\frac{\varepsilon}{2},
$$

where $J_{k} g$ is the $k$-dimensional Jacobian of $g$. As a consequence, it will be sufficient to find a continuous homotopy map $H:[0,1] \times \partial Q \rightarrow \mathbf{R}^{n+N}$ from $(I d \bowtie v)_{\mid \partial Q}$ to a Lipschitz map $f$

$$
H(0, x)=(I d \bowtie v)(x), \quad H(1, x)=f(x), \quad x \in \partial Q
$$

such that $H$ is in $\operatorname{cart}^{1}\left([0,1] \times \partial Q, \mathbf{R}^{n+N}\right)$, with boundary given by the homotopy formula [16, 26.22]

$$
\begin{aligned}
\partial H_{\#}(\llbracket 0,1 \rrbracket \times \llbracket \partial Q \rrbracket) & =H_{\#}\left(\delta_{1} \times \llbracket \partial Q \rrbracket\right)-H_{\#}\left(\delta_{0} \times \llbracket \partial Q \rrbracket\right) \\
& =f_{\#} \llbracket \partial Q \rrbracket-(I d \bowtie v)_{\#} \llbracket \partial Q \rrbracket
\end{aligned}
$$

and with $k$-dimensional mapping area less than $\varepsilon / 2$. In fact, taking

$$
\widetilde{T}:=T+H_{\#}(\llbracket 0,1 \rrbracket \times \llbracket \partial Q \rrbracket)
$$

and observing that $\partial \widetilde{T}=f_{\#} \llbracket \partial Q \rrbracket$ has to hold by (9) and (10), the map $w$ can be defined as follows

$$
w(x):=\left\{\begin{array}{lll}
\pi \circ g(2 x) & \text { if } & \|x\| \leq 1 / 2 \\
\pi \circ H\left(2-2\|x\|, \frac{x}{\|x\|}\right) & \text { if } & 1 / 2 \leq\|x\| \leq 1
\end{array}\right.
$$


where $\|x\|:=\max _{1 \leq i \leq k}\left|x_{i}\right|$ and $\pi: \mathbf{R}^{n} \times \mathbf{R}^{N} \rightarrow \mathbf{R}^{N}$ is the orthogonal projection onto the last $N$ coordinates.

To define $H$, denote by $\mathcal{L}_{j}$ the $j$-dimensional skeleton of the grid $\mathcal{L}$ of mesh $2 \delta$ in $\mathbf{R}^{n+N}$

$$
\mathcal{L}=\mathcal{L}(2 \delta):=\bigcup_{z \in \mathbf{Z}^{n+N}} \delta\left(z+[-1,1]^{n+N}\right)
$$

Similarly to [16, Sect. 29], we can define a deformation map $\Psi$ of $\mathbf{R}^{n+N}$ onto the $(k-1)$-skeleton $\mathcal{L}_{k-1}$ such that $\Psi$ is Lipschitz continuous on $(I d \bowtie v)(\partial Q)$ (since $v$ is continuous on $\partial Q$ ) and such that if $h:[0,1] \times \partial Q \rightarrow \mathbf{R}^{n+N}$ is the affine homotopy between $I d \bowtie v$ and $\Psi \circ(I d \bowtie v)$

$$
h(t, x):=t \cdot \Psi \circ(I d \bowtie v)(x)+(1-t) \cdot(I d \bowtie v)(x), \quad 0 \leq t \leq 1, \quad x \in \partial Q
$$

then the $k$-dimensional mapping area of $h$ is small with $\delta$. Also, by means of compositions of central projections from each $j$-face of the skeleton $\mathcal{L}_{j}$ onto its boundary, for decreasing $j$ from $k-1$ to 1 , one can assume that the map

$$
\tilde{f}:=\Psi \circ(I d \bowtie v): \partial Q \longrightarrow \mathcal{L}_{k-1}
$$

takes each $j$-face $F$ of $Q_{(j)}$ onto the $j$-skeleton $\mathcal{L}_{j}$ for all $j=0, \ldots, k-1$, whereas (for $j \geq 1$ )

$$
\int_{(I d \bowtie v)(F)} J_{j} \Psi d \mathcal{H}^{j} \leq c\left[\mathbf { M } \left(G_{v_{\mid F}}\left\llcorner F \times \mathbf{R}^{N}\right)+\delta \mathbf{M}\left(\partial\left(G_{v_{\mid F}}\left\llcorner F \times \mathbf{R}^{N}\right)\right)\right]\right.\right.
$$

for some absolute constant $c>0$, where $J_{j} \Psi$ is the $j$-dimensional Jacobian of the restriction of $\Psi$ to $(I d \bowtie v)(F)$ and $v_{\mid F}$ is the restriction of $v$ to $F$ (hence $G_{v_{\mid F}}$ is $j$-dimensional). Note that the right-hand side of (11) is finite by the generic position of $v$ w.r.t. $\partial Q$.

Moreover, by (11) it can be defined a continuous homotopy map $\widetilde{h}:[0,1] \times$ $\partial Q \rightarrow \mathcal{L}_{k-1}$ from $\widetilde{f}$ to a Lipschitz map $f: \partial Q \rightarrow \mathcal{L}_{k-1}$

$$
\widetilde{h}(0, x)=\widetilde{f}(x), \quad \widetilde{h}(1, x)=f(x), \quad x \in \partial Q
$$

such that $\widetilde{h}$ is in $\operatorname{cart}^{1}\left([0,1] \times \partial Q, \mathbf{R}^{n+N}\right)$ and $\widetilde{h}$ has zero $k$-dimensional mapping area, since its image is contained in $\mathcal{L}_{k-1}$. Indeed, for all $j=1, \ldots, k-1$ and for all $j$-faces $F$ of $Q_{(j)}$, we can define a continuous homotopy map $\widetilde{h}_{F}:[0,1] \times F \rightarrow \mathcal{L}_{j}$ from $\widetilde{f}_{\mid F}: F \rightarrow \mathcal{L}_{j}$ to a Lipschitz map (by (11)) $f_{F}: F \rightarrow \mathcal{L}_{j}$ such that $\widetilde{h}_{F}([0,1] \times F) \subset \widetilde{f}(F), \widetilde{h}_{F}$ is in $\operatorname{cart}^{1}\left([0,1] \times F, \mathbf{R}^{n+N}\right)$ and

(a) if $j=1, \widetilde{h}_{F}$ is constant on $[0,1] \times \partial F$;

(b) if $j \geq 2$, for each $(j-1)$-face $I$ of the boundary of $F$, the restriction of $\widetilde{h}_{F}$ to $[0,1] \times I$ is equal to the homotopy $\widetilde{h}_{I}:[0,1] \times I \rightarrow \mathcal{L}_{j-1}$ defined at the previous step, with boundary

$$
\begin{aligned}
\partial\left(\widetilde{h}_{F \#}(\llbracket 0,1 \rrbracket \times \llbracket F \rrbracket)\right) & =\widetilde{h}_{F \#}\left(\delta_{1} \times \llbracket F \rrbracket\right)-\widetilde{h}_{F \#}\left(\delta_{0} \times \llbracket F \rrbracket\right) \\
& -\sum_{\substack{I \in Q_{(j-1)} \\
I \subset \partial F}} \sigma_{I} \widetilde{h}_{I \#}(\llbracket 0,1 \rrbracket \times \llbracket I \rrbracket)
\end{aligned}
$$


if $\llbracket \partial F \rrbracket=\sum\left\{\sigma_{I} \llbracket I \rrbracket \mid I \in Q_{(j-1)}, I \subset \partial F\right\}$ for suitable $\sigma_{I}= \pm 1, \llbracket F \rrbracket$ and $\llbracket I \rrbracket$ being equipped with the natural orientations.

Hence, there is no creation of boundary or discontinuities when defining $\widetilde{h}$, as required. Finally take

$$
H(t, x):=\left\{\begin{array}{lll}
h(2 t, x) & \text { if } \quad 0 \leq t \leq 1 / 2, & x \in \partial Q \\
\widetilde{h}(2 t-1, x) & \text { if } \quad 1 / 2 \leq t \leq 1, & x \in \partial Q
\end{array}\right.
$$

\section{Local properties of 2-dimensional approximable graphs}

Let $u$ be a vector valued map in $\operatorname{cart}^{1}\left(\Omega, \mathbf{R}^{N}\right)$, defined on a bounded 2-dimensional domain $\Omega \subset \mathbf{R}^{2}$, which can be approximated strongly in $L^{1}$ with all minors by smooth maps $u_{k}: \Omega \rightarrow \mathbf{R}^{N}$, i.e., such that (2) holds for smooth $u_{k}$ 's. Consider a point $x_{0} \in \Omega$, without loss of generality $x_{0}=0_{\mathbf{R}^{2}}$. By the coarea formula [3, 3.2.11], for $\mathcal{H}^{1}$-a.e. $0<\rho<\widetilde{\rho}, \widetilde{\rho}:=\operatorname{dist}\left(0_{\mathbf{R}^{2}}, \partial \Omega\right)$, (2) yields

$$
\lim _{k \rightarrow+\infty} \int_{\partial B_{\rho}^{2}}\left(\left|u_{k}-u\right|+\left|D u_{k}-D u\right|\right) d \mathcal{H}^{1}=0,
$$

with $u_{\mid \partial B_{\rho}^{2}}$ absolutely continuous. In particular, by a.e. convergence $u_{k} \rightarrow u$ in $\partial B_{\rho}^{2}$, and by equi-integrability of $\left\{D u_{\left.k \mid \partial B_{\rho}^{2}\right\}}\right.$, one has

$$
\lim _{k \rightarrow+\infty}\left\|u_{k}-u\right\|_{\infty, \partial B_{\rho}^{2}}=0
$$

uniformly in $C^{0}$.

Denote now by $h_{k}:[0,1] \times \partial B_{\rho}^{2} \rightarrow \mathbf{R}^{N}$ the affine homotopy map between the traces of $u$ and $u_{k}$ over the boundary $\partial B_{\rho}^{2}$, i.e.,

$$
h_{k}(t, x):=t u_{k}(x)+(1-t) u(x), \quad 0 \leq t \leq 1, \quad x \in \partial B_{\rho}^{2} .
$$

Also, for $k>1 / \rho$, denote by $w_{k}: B_{\rho}^{2} \rightarrow \mathbf{R}^{N}$ the map

$$
w_{k}(x):=\left\{\begin{array}{lll}
u_{k}\left(\frac{k \rho}{k \rho-1} x\right) & \text { if } & |x| \leq \rho-1 / k \\
h_{k}\left(k(\rho-|x|), \rho \frac{x}{|x|}\right) & \text { if } & \rho-1 / k \leq|x| \leq \rho .
\end{array}\right.
$$

With the previous notations we have

Proposition 2. The following two facts hold:

i) for any $k \in \mathbf{N}, k>1 / \rho$, the map $w_{k}$ is in $\operatorname{cart}^{1}\left(B_{\rho}^{2}, \mathbf{R}^{N}\right)$, continuous up to the closure of $B_{\rho}^{2}$ and with boundary

$$
\partial\left(G_{w_{k}}\left\llcorner B_{\rho}^{2} \times \mathbf{R}^{N}\right)=\partial\left(G_{u}\left\llcorner B_{\rho}^{2} \times \mathbf{R}^{N}\right)\right.\right.
$$


in particular $w_{k \mid \partial B_{\rho}^{2}}=u_{\mid \partial B_{\rho}^{2}}$. Moreover, for each small $\eta>0$

$$
\int_{B_{\rho}^{2}}\left|M\left(D w_{k}\right)\right| d x \leq \int_{B_{\rho}^{2}}|M(D u)| d x+\eta
$$

for $k$ sufficiently large, where $|M(D v)|$ denotes the 2-dimensional Jacobian of Id $\bowtie v$, for $v=u, w_{k}$;

ii) in case of codimension $N \geq 3$, if

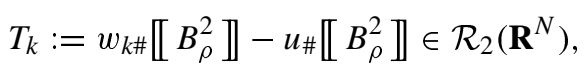

there exists an i.m. rectifiable current $R_{k} \in \mathcal{R}_{3}\left(\mathbf{R}^{N}\right)$ with boundary $T_{k}$, $\partial R_{k}=T_{k}$, and arbitrary small mass, i.e.,

$$
\mathbf{M}\left(R_{k}\right) \leq \eta
$$

for $k$ sufficiently large.

Proof. To show (15), by changing variables and using (2) we infer

$$
\int_{B_{\rho-1 / k}^{2}}\left|M\left(D w_{k}\right)\right| d x \leq \int_{B_{\rho}^{2}}\left|M\left(D u_{k}\right)\right| d x \leq \int_{B_{\rho}^{2}}|M(D u)| d x+\eta
$$

for $k$ large. Moreover, if $\left|M_{(2)}\left(D h_{k}\right)\right|$ denotes the 2-dimensional Jacobian of the differential of $h_{k}$ w.r.t. $(t, \tau)$, where $\tau$ is the tangential direction to $\partial B_{\rho}^{2}$, since $h_{k} \in \operatorname{cart}^{1}\left([0,1] \times \partial B_{\rho}^{2}, \mathbf{R}^{N}\right)$, we have

$$
\begin{aligned}
& \int_{B_{\rho}^{2} \backslash B_{\rho-1 / k}^{2}}\left|M\left(D w_{k}\right)\right| d x \leq \\
& \quad \leq \int_{0}^{1} d t \int_{\partial B_{\rho}^{2}} \sqrt{\left|u_{k}-u\right|^{2}+\left|M_{(2)}\left(D h_{k}\right)\right|^{2}} d \mathcal{H}^{1}+O(1 / k)
\end{aligned}
$$

which is small by (12) and (13), for $k$ large, since for a.e. $(t, x) \in[0,1] \times \partial B_{\rho}^{2}$

$$
\left|M_{(2)}\left(D h_{k}(t, x)\right)\right| \leq\left|u_{k}(x)-u(x)\right|\left|t D_{\tau} u_{k}(x)+(1-t) D_{\tau} u(x)\right| .
$$

Then (18) and (19) yield (15), whereas (14) holds since by the homotopy formula and (8)

$$
\begin{aligned}
\partial h_{k \#}\left(\llbracket 0,1 \rrbracket \times \llbracket \partial B_{\rho}^{2} \rrbracket\right) & =u_{k \mid \partial B_{\rho}^{2 \#} \llbracket} \llbracket \partial B_{\rho} \rrbracket-u_{\mid \partial B_{\rho}^{2} \# \llbracket \partial B_{\rho}^{2} \rrbracket} \\
& =\partial u_{k \#} \llbracket B_{\rho}^{2} \rrbracket-\partial u_{\sharp} \llbracket B_{\rho}^{2} \rrbracket
\end{aligned}
$$

and then $w$ is in $\operatorname{cart}^{1}\left(B_{\rho}^{2}, \mathbf{R}^{N}\right)$ with boundary

$$
\begin{aligned}
\partial\left(G_{w_{k}}\left\llcorner B_{\rho}^{2} \times \mathbf{R}^{N}\right)\right. & =\left(I d \bowtie w_{k}\right)_{\mid \partial B_{\rho}^{2 \#} \llbracket}\left[\partial B_{\rho}^{2} \rrbracket\right] \\
& =(I d \bowtie u)_{\mid \partial B_{\rho}^{2 \#}}\left[\partial B_{\rho}^{2} \rrbracket .\right.
\end{aligned}
$$


Finally, to show (17) we make use of an extension of [16, 31.2], stating equivalence of weak convergence and flat metric convergence for i.m. rectifiable currents with equibounded mass and boundary mass. To this aim, fix $M>0$ and an increasing sequence $\left\{r_{j}\right\}$ of positive radii. For $r>0$ denote by $C_{r}$ the complement of the $N$-cube of side $2 r$ of $\mathbf{R}^{N}$

$$
C_{r}:=\left\{y=\left(y_{1}, \ldots, y_{N}\right) \in \mathbf{R}^{N}\left|\max _{1 \leq i \leq N}\right| y_{i} \mid>r\right\}
$$

Also set

$$
\mathcal{I}:=\left\{T \in \mathcal{R}_{2}\left(\mathbf{R}^{N}\right) \mid \mathbf{M}(T) \leq M, \quad \partial T=0, \quad \mathbf{M}\left(T\left\llcorner C_{r_{j}}\right) \leq 1 / j \quad \forall j \in \mathbf{N}\right\}\right.
$$

and for $T_{1}, T_{2} \in \mathcal{R}_{2}\left(\mathbf{R}^{N}\right)$, with $\partial T_{1}=\partial T_{2}=0$, define the metric

$$
d\left(T_{1}, T_{2}\right):=\inf \left\{\mathbf{M}(R) \mid R \in \mathcal{R}_{3}\left(\mathbf{R}^{N}\right), \quad \partial R=T_{1}-T_{2}\right\}
$$

Lemma 1. If $\left\{T_{k}\right\}, T \in \mathcal{I}$, then $T_{k} \rightarrow T$ weakly in $\mathcal{D}_{2}\left(\mathbf{R}^{N}\right)$ if and only if $d\left(T_{k}, T\right) \rightarrow 0$ as $k \rightarrow+\infty$.

To prove (17), it then suffices to show that $\left\{T_{k}\right\} \in \mathcal{I}$. In fact, by (16) and (15), with $\eta=1$,

$$
\mathbf{M}\left(T_{k}\right) \leq 2 \mathbf{M}\left(G_{u}\left\llcorner B_{\rho}^{2} \times \mathbf{R}^{N}\right)+1=M\right.
$$

for $k$ sufficiently large, whereas by (14) and (8) $\partial T_{k}=0$. Also, by $L^{1}$-convergence of $w_{k}$ to $u$ we have

$$
\sup _{k \in \mathbf{N}} \operatorname{meas}\left(\left\{x \in B_{\rho}^{2}|| w_{k}(x) \mid>r\right\}\right) \rightarrow 0
$$

as $r \rightarrow+\infty$, thus by equi-integrability we infer, for each small $\varepsilon>0$,

$$
\sup _{k \in \mathbf{N}} \int_{B_{\rho}^{2} \cap\left\{\left|w_{k}(x)\right|>r\right\}}\left|M_{(2)}\left(D w_{k}\right)\right| d x \leq \varepsilon
$$

for $r \geq r(\varepsilon)$. Then, for a suitable increasing sequence $\left\{r_{j}\right\}$, for each $j \in \mathbf{N}$ we have $\sup _{k \in \mathbf{N}} \mathbf{M}\left(T_{k}\left\llcorner C_{r_{j}}\right) \leq 1 / j\right.$, hence $\left\{T_{k}\right\} \in \mathcal{I}$. Therefore, since by (18) and (19) we have $T_{k} \rightarrow 0$ as $k \rightarrow+\infty$, by Lemma 1 we obtain $R_{k} \in \mathcal{R}_{3}\left(\mathbf{R}^{N}\right)$ with boundary $\partial R_{k}=T_{k}$ and for which (17) holds.

We end this section with the following

Proof of Lemma 1. The "if" part is trivial. To show the "only if" part, we claim that $\mathcal{I}$ is totally bounded w.r.t. the metric $d$. More precisely, for each small $\varepsilon>0$, we can find $m=m(\varepsilon) \in \mathbf{N}$ and $P_{i} \in \mathcal{R}_{2}\left(\mathbf{R}^{N}\right), i=1, \ldots, m$, with $\partial P_{i}=0$ and 
such that

$$
\mathcal{I} \subset \sum_{i=1}^{m}\left\{S \in \mathcal{I} \mid d\left(S, P_{i}\right) \leq \varepsilon\right\} .
$$

In fact, by the deformation theorem [16, 29.3], for fixed $\delta>0$, to each $T \in \mathcal{I}$ it corresponds an integral polyhedral 2-chain $P$, with zero boundary $\partial P=0$, supported in the 2-skeleton $\mathcal{L}_{2}(\delta)$ of the grid of mesh $\delta$ in $\mathbf{R}^{N}$

$$
\mathcal{L}(\delta):=\bigcup_{z \in \mathbf{Z}^{N}} \delta\left(z+[0,1]^{N}\right)
$$

and an i.m. rectifiable current $R \in \mathcal{R}_{3}\left(\mathbf{R}^{N}\right)$ such that

$$
T=P+\partial R
$$

with mass estimates

$$
\mathbf{M}(P) \leq c \mathbf{M}(T), \quad \mathbf{M}(R) \leq c \delta \mathbf{M}(T)
$$

for some absolute constant $c>0$. If $\delta>0$ is chosen sufficiently small we have

$$
\mathbf{M}(R) \leq c \delta M \leq \varepsilon .
$$

Suppose now $P$ is given by

$$
P=\sum_{F \in \mathcal{L}_{2}(\delta)} \beta_{F} \llbracket F \rrbracket, \quad \beta_{F} \in \mathbf{Z} .
$$

Making use of the deformation technique due to Almgren [2, 1.15], one also obtains the following local mass estimates for $P$

$$
\left|\beta_{F}\right| \delta^{2}=\mathbf{M}(P\llcorner F) \leq c \mathbf{M}(T\llcorner\cup \operatorname{nbs}(F))
$$

for each 2-face $F \in \mathcal{L}_{2}(\delta)$. Here $\operatorname{nbs}(F)$ is the family of $N$-cubes of the grid $\mathcal{L}(\delta)$ which contain $F$ in their boundary. Clearly, if $F \subset C_{r}$, then $\cup \operatorname{nbs}(F) \subset C_{r-\delta}$, so that if $r=r_{j}+\delta$ for some $j \in \mathbf{N}$, one has

$$
\mathbf{M}\left(T\llcorner\cup \operatorname{nbs}(F)) \leq \mathbf{M}\left(T\left\llcorner C_{r_{j}}\right) \leq 1 / j .\right.\right.
$$

Hence, if $j>c \delta^{-2}$, with $c>0$ given by (20), by (21) we infer $\beta_{F}=0$ for each $F \subset C_{r}$ and therefore spt $P \cap C_{r}=\emptyset$. Since there is only a finite number of polyhedral 2-chains $P$ of $\mathbf{R}^{N}$ with boundary $\partial P=0$, with mass $\mathbf{M}(P) \leq c M$ and support

$$
\text { spt } P \subset \bigcup\left\{F \in \mathcal{L}_{2}(\delta) \mid F \subset \mathbf{R}^{N} \backslash C_{r}\right\},
$$

the total boundedness property holds. The rest of the proof is similar to $[16,31.2]$. 


\section{Local approximation argument}

Let now $u$ be a map in $\operatorname{cart}^{1}\left(\Omega, \mathbf{R}^{N}\right)$ such that for a.e. 2-plane intersecting the domain the restriction of $u$ can be approximated strongly in $L^{1}$ with all minors by smooth maps, according to the main result.

In this section we describe how to substitute the graph of $u$ over small pieces of the domain $\Omega$ by the graph of a nice map $v$, without creating discontinuities and with a suitable control of the area, compare (25).

To this aim, following the notations in Sect. 1, let $Q:=[-\delta, \delta]^{n}$, for fixed small $0<\delta<1$, suppose $Q$ is strictly contained in $\Omega$ and in generic position w.r.t. $u$, compare Definition 2 in Sect. 1 . Then, the restriction $u_{\mid K}$ is in $\operatorname{cart}^{1}\left(K, \mathbf{R}^{N}\right)$ for each $k$-face $K$ of $Q_{(k)}, k=1, \ldots, n-1$.

By an easy generalization of Proposition 2 in Sect. 2, up to a slight displacement of the faces of the cube $Q$, one infers that for each 2-face $F$ of $Q_{(2)}$, denoting by $u_{F}:=u_{\mid F}$ the restriction of $u$ to $F$, for each small $\eta>0$ the following conditions hold:

i) there exists a map $v_{F}: F \rightarrow \mathbf{R}^{N}$ in $\operatorname{cart}^{1}\left(F, \mathbf{R}^{N}\right)$, continuous up to the closure of $F$, such that

$$
\partial\left(G_{v_{F}}\left\llcorner F \times \mathbf{R}^{N}\right)=\partial\left(G_{u_{F}}\left\llcorner F \times \mathbf{R}^{N}\right),\right.\right.
$$

in particular $v_{F \mid \partial F}=u_{F \mid \partial F}$, and

$$
\int_{F}\left|M\left(D v_{F}\right)\right| d \mathcal{H}^{2} \leq \int_{F}\left|M\left(D u_{F}\right)\right| d \mathcal{H}^{2}+\eta
$$

ii) in case of codimension $N \geq 3$, there exists an i.m. rectifiable current $R_{F} \in$ $\mathcal{R}_{3}\left(\mathbf{R}^{N}\right)$ with boundary

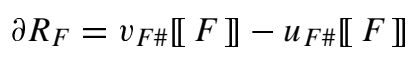

and small mass

$$
\mathbf{M}\left(R_{F}\right) \leq \eta
$$

Remark 2. In (22), $\left|M\left(D v_{F}\right)\right|$ denotes the 2-dimensional Jacobian of the differential of the join map $I d \bowtie v_{F}$ (the same for $u_{F}$ ), see Definition 1. Moreover, the i.m. rectifiable current $\llbracket F \rrbracket \in \mathcal{R}_{2}\left(\mathbf{R}^{n}\right)$ is equipped with the natural orientation induced by the canonical basis of $\mathbf{R}^{n}$. Also, (23) makes sense since $u_{F}$ and $v_{F}$ are Cartesian maps with the same boundary, thus by (8)

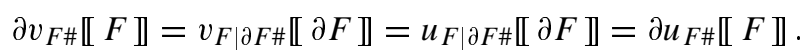

We show that to define a suitable approximating map for $u$ on $Q$, it suffices, by i), to substitute $u$ on the 2 -faces of $Q$ and, by ii), to fill the "holes" this way produced. In fact, thanks to the previous hypotheses i) and ii), and to the generic position of $Q$ w.r.t. $u$, we infer the following 
Proposition 3. For each small $\varepsilon>0$ there exists a map $v: Q \rightarrow \mathbf{R}^{N}$ in $\operatorname{cart}^{1}\left(Q, \mathbf{R}^{N}\right)$, continuous up to the closure of $Q$, such that the restriction of $v$ to each $(n-j)$-face $K$ of $Q_{(n-j)}, j=1, \ldots, n-2$, is a map in $\operatorname{cart}^{1}\left(K, \mathbf{R}^{N}\right)$ which only depends on the restriction $u_{K}:=u_{\mid K}$ of $u$ to $K$. Moreover, $v$ coincides with $u$ on the 1-skeleton $Q_{(1)}$ of $Q$ and the following integral estimate holds

$$
\begin{aligned}
\mathbf{M}\left(G_{v}\left\llcorner Q \times \mathbf{R}^{N}\right)\right. & \leq \mathbf{M}\left(G_{u}\left\llcorner Q \times \mathbf{R}^{N}\right)+\varepsilon\right. \\
& +c \sum_{j=1}^{n-2} \delta^{j} \sum_{K \in Q_{(n-j)}} \int_{K}\left|M\left(D u_{K}\right)\right| d \mathcal{H}^{n-j}
\end{aligned}
$$

where $c>0$ is an absolute constant.

Remark 3. It comes out from the proof (see Proposition 1 in Sect. 1 , case $N<k$ ) that, in case $N<n,(25)$ can be improved by taking off the term $\mathbf{M}\left(G_{u}\left\llcorner Q \times \mathbf{R}^{N}\right)\right.$ and by limiting the summation to $j=n-N, \ldots, n-2$.

Proof of Proposition 3. For fixed $0<\lambda<1$, denote by $\lambda Q:=[-\lambda \delta,+\lambda \delta]^{n}$ the $\lambda$-homothetic of the $n$-cube $Q=[-\delta, \delta]^{n}$. Also, denote by $\Sigma_{(k)}$, for $k=1, \ldots, n$, the $k$-dimensional subset of $Q \backslash \operatorname{int}(\lambda Q)$ given by the union of the convex hull of the corresponding $(k-1)$-faces of $Q_{(k-1)}$ and $\lambda Q_{(k-1)}$, i.e.,

$$
\Sigma_{(k)}:=\bigcup_{F \in Q_{(k-1)}} \operatorname{co}(F \cup \lambda F)=\left\{t x \mid \lambda \leq t \leq 1, \quad x \in Q_{(k-1)}\right\},
$$

so that $Q_{(n)}=Q, Q_{(n-1)}=\partial Q$ and $\Sigma_{(n)}=Q \backslash \operatorname{int}(\lambda Q)$.

The proof divides in $n$ parts. At the $k^{\text {th }}$ step, for $k=1, \ldots, n$, we define $v$ on the $k$-dimensional set $\Sigma_{(k)} \cup Q_{(k)}$, making use of the restriction of $u$ to $Q_{(k)}$ and of the definition of $v$ at the previous step. The argument is a generalization of the case of dimension $n=3$, so that we address the reader to the case $n=3$ of $3^{\text {rd }}$ step for a better understanding of the proof.

$1^{\text {st }}$ step: definition of $v$ on $\Sigma_{(1)} \cup Q_{(1)}$.

Define $v$ on $\Sigma_{(1)}$ by taking

$$
v(t x)=u(x), \quad \lambda \leq t \leq 1, \quad x \in Q_{(0)}
$$

and on $Q_{(1)}$ by

$$
v(x)=u(x), \quad x \in Q_{(1)} .
$$

$2^{\text {nd }}$ step: definition of $v$ on $\Sigma_{(2)} \cup Q_{(2)}$.

Define $v$ on $\Sigma_{(2)}$ by taking

$$
v(t x)=u(x), \quad \lambda \leq t \leq 1, \quad x \in Q_{(1)} .
$$

To define $v$ on $Q_{(2)}$, for each 2-face $F$ of $Q_{(2)}$ take

$$
v(x)=v_{F}(x), \quad x \in F
$$

where $v_{F}: F \rightarrow \mathbf{R}^{N}$ is the continuous map in $\operatorname{cart}^{1}\left(F, \mathbf{R}^{N}\right)$ given by remark i) at the beginning of this section, so that (22) holds. 
Remark 4. Note that if $F_{1}, F_{2} \in Q_{(2)}$ are such that $\partial F_{1} \cap \partial F_{2}=I \in Q_{(1)}$, and $v_{\mid F}=v_{F}$, then one has

$$
\partial\left(G _ { v _ { | F _ { 1 } } } \llcorner \operatorname { i n t } ( F _ { 1 } ) \times \mathbf { R } ^ { N } ) \left\llcornerI \times \mathbf{R}^{N}=-\partial\left(G _ { v _ { | F _ { 2 } } } \llcorner \operatorname { i n t } ( F _ { 2 } ) \times \mathbf { R } ^ { N } ) \left\llcornerI \times \mathbf{R}^{N} .\right.\right.\right.\right.
$$

$3^{\text {rd }}$ step: definition of $v$ on $\Sigma_{(3)} \cup Q_{(3)}$.

In the sequel, in order to simplify computations, we will often define $v$ by means of implicit maps $G$, so that $G(z)=(\varphi(z), v(\varphi(z)))$ for some diffeomorphism $x=\varphi(z)$ of $z \in D$ onto $x \in \Delta$, where $D, \Delta$ are $k$-dimensional. In fact, since the area functional does not depend on the parametrization, by the area formula $[3$, 3.2.3] we have

$$
\int_{\Delta}|M(D v(x))| d \mathcal{H}^{k}(x)=\int_{D} J_{k} G(z) d \mathcal{H}^{k}(z),
$$

where $J_{k} G$ is the $k$-dimensional Jacobian of $G$.

To define $v$ on $\Sigma_{(3)}$, consider a 2 -face $F$ of $Q_{(2)}$, for simplicity

$$
F=\left\{x \in \mathbf{R}^{n} \mid x=(\widetilde{x}, \widehat{\delta}),\|\widetilde{x}\|_{2} \leq \delta\right\} .
$$

Here we denote $\tilde{x}:=\left(x_{1}, x_{2}\right) \in \mathbf{R}^{2}, \widehat{t}:=(t, \ldots, t) \in \mathbf{R}^{n-2}$ if $t \in \mathbf{R},\|\widetilde{x}\|_{2}:=$ $\max _{1 \leq i \leq 2}\left|x_{i}\right|$. We will define $v$ on the convex hull

$$
\operatorname{co}(F \cup \lambda F)=\left\{(\tilde{x}, \widehat{t}) \mid \lambda \delta \leq t \leq \delta, \quad\|\tilde{x}\|_{2} \leq t\right\}
$$

by using the maps $u_{F}:=u_{\mid F}$ and $v_{F}$, see $2^{\text {nd }}$ step.

To this aim divide in three parts

$$
\operatorname{co}(F \cup \lambda F)=\bigcup_{i=1}^{3} \Delta_{F}^{i}
$$

and define $v$ on the $\Delta_{F}^{i}$,s by means of implicit maps $G^{(i)}$. Set

$$
\Delta_{F}^{1}:=\left\{(\tilde{x}, \widehat{t}) \mid \delta\left(\frac{\lambda+1}{2}\right)<t \leq \delta, \quad\|\widetilde{x}\|_{2} \leq \delta f(t)\right\}
$$

where $f:\left[\delta\left(\frac{\lambda+1}{2}\right), \delta\right] \rightarrow[0,1]$ is the affine map such that $f\left(\delta\left(\frac{\lambda+1}{2}\right)\right)=0$, $f(\delta)=1$, i.e.,

$$
f(t):=\frac{1}{\lambda-1}\left(\lambda+1-\frac{2}{\delta} t\right) .
$$

Moreover, let us define $v$ on $\Delta_{F}^{1}$ by means of the implicit map

$$
\begin{gathered}
\left.\left.G^{(1)}:\right] \delta\left(\frac{\lambda+1}{2}\right), \delta\right] \times[-\delta, \delta]^{2} \longrightarrow \Delta_{F}^{1} \times \mathbf{R}^{N} \\
G^{(1)}(t, \tilde{x}):=\left((f(t) \tilde{x}, \widehat{t}), v_{F}(\tilde{x}, \widehat{\delta})\right) .
\end{gathered}
$$


We then infer

$$
\int_{\Delta_{F}^{1}}|M(D v)| d \mathcal{H}^{3} \leq \sqrt{2} \delta \int_{F}\left|M\left(D v_{F}\right)\right| d \mathcal{H}^{2}+O(1-\lambda) .
$$

In fact, as previously remarked, by the area formula we get

$$
\int_{\Delta_{F}^{1}}|M(D v)| d \mathcal{H}^{3}=\int_{\delta\left(\frac{\lambda+1}{2}\right)}^{\delta} d t \int_{[-\delta, \delta]^{2}} J_{3} G^{(1)} d \mathcal{H}^{2}
$$

where $J_{3} G^{(1)}$ is the 3-dimensional Jacobian of $G^{(1)}$. By direct computation

$$
\begin{aligned}
\left(J_{3} G^{(1)}\right)^{2} & =(n-2) f(t)^{4}+\left(f(t) \frac{2}{\delta(1-\lambda)}<\tilde{x}, D v_{F}>\right)^{2} \\
& +(n-2) f(t)^{2}\left|D v_{F}\right|^{2}+\left(\frac{2}{\delta(1-\lambda)}\right)^{2}\left(\left(x_{1}\right)^{2}+\left(x_{2}\right)^{2}\right)\left|M_{(2)}\left(D v_{F}\right)\right|^{2} \\
& +(n-2)\left|M_{(2)}\left(D v_{F}\right)\right|^{2} .
\end{aligned}
$$

Since $\left|<\tilde{x}, D v_{F}>\right|^{2} \leq\left(\left(x_{1}\right)^{2}+\left(x_{2}\right)^{2}\right)\left|D v_{F}\right|^{2}$, for $\lambda$ close to 1 we obtain (28). Set

$$
\Delta_{F}^{2}:=\left\{(\tilde{x}, \widehat{t}) \mid \delta \lambda \leq t<\delta\left(\frac{\lambda+1}{2}\right), \quad\|\tilde{x}\|_{2} \leq \delta f(t)\right\}
$$

where $f:\left[\delta \lambda, \delta\left(\frac{\lambda+1}{2}\right)\right] \rightarrow[0, \lambda]$ is the affine map such that $f(\delta \lambda)=\lambda$ and $f\left(\delta\left(\frac{\lambda+1}{2}\right)\right)=0$, i.e.,

$$
f(t):=\frac{\lambda}{\delta(1-\lambda)}(\delta(\lambda+1)-2 t) .
$$

Moreover, let us define $v$ on $\Delta_{F}^{2}$ by means of the implicit map

$$
\begin{gathered}
G^{(2)}:\left[\delta \lambda, \delta\left(\frac{\lambda+1}{2}\right)\left[\times[-\delta, \delta]^{2} \longrightarrow \Delta_{F}^{2} \times \mathbf{R}^{N}\right.\right. \\
G^{(2)}(t, \tilde{x}):=\left((f(t) \tilde{x}, \widehat{t}), u_{F}(\tilde{x}, \widehat{\delta})\right) .
\end{gathered}
$$

By a computation similar to the one above, we obtain

$$
\int_{\Delta_{F}^{2}}|M(D v)| d \mathcal{H}^{3} \leq \sqrt{2} \delta \int_{F}\left|M\left(D u_{F}\right)\right| d \mathcal{H}^{2}+O(1-\lambda) .
$$

Set

$$
\Delta_{F}^{3}:=\left\{(\widetilde{x}, \widehat{t}) \mid \delta \lambda \leq t \leq \delta, \quad \delta f(t)<\|\widetilde{x}\|_{2} \leq t\right\}
$$

where $f:[\delta \lambda, \delta] \rightarrow[0, \lambda]$ is the gluing of the previous maps

$$
f(t):=\left\{\begin{array}{lll}
\frac{\lambda}{\delta(1-\lambda)}(\delta(\lambda+1)-2 t) & \text { if } & \delta \lambda \leq t \leq \delta\left(\frac{\lambda+1}{2}\right) \\
\frac{1}{\lambda-1}\left(\lambda+1-\frac{2}{\delta} t\right) & \text { if } \quad & \delta\left(\frac{\lambda+1}{2}\right) \leq t \leq \delta .
\end{array}\right.
$$


Moreover, define $v$ on $\Delta_{F}^{3}$ by means of the trace of $u$ on the boundary of $F$, as follows. For fixed $(\widetilde{x}, \widehat{\delta}) \in \partial F$, so that $\|\widetilde{x}\|_{2}=\delta$, and for each $\left.\left.\sigma \in\right] 0,1\right], v$ takes the constant value $u(\widetilde{x}, \widehat{\delta})$ on the parallel line segments connecting the points $\left(\lambda \sigma \widetilde{x}, \frac{1}{2}[(1+\lambda)+\sigma(\lambda-1)] \widehat{\delta}\right)$ and $\left(\sigma \widetilde{x}, \frac{1}{2}[(1+\lambda)+\sigma(1-\lambda)] \widehat{\delta}\right)$. More precisely, $v$ is defined by the implicit map

$$
\begin{gathered}
\left.\left.G^{(3)}:\left\{\tilde{x} \in \mathbf{R}^{2} \mid\|\tilde{x}\|_{2}=\delta\right\} \times[\lambda, 1] \times\right] 0,1\right] \longrightarrow \Delta_{F}^{3} \times \mathbf{R}^{N} \\
G^{(3)}(\tilde{x}, t, \sigma):=\left(\left(t \sigma \tilde{x}, \frac{1}{2}[(1+\lambda)+\sigma(2 t-(1+\lambda))] \widehat{\delta}\right), u(\tilde{x}, \widehat{\delta})\right) .
\end{gathered}
$$

Denoting by $u_{I}$ the restriction of $u$ to the 1 -face $I$ of the boundary of $F$, one easily infers

$$
\int_{\Delta_{F}^{3}}|M(D v)| d \mathcal{H}^{3} \leq c \delta^{2}(1-\lambda) \sum_{\substack{I \in Q_{(1)} \\ I \subset \partial F}} \int_{I}\left|M\left(D u_{I}\right)\right| d \mathcal{H}^{1}
$$

for some absolute constant $c>0$. Therefore, the second member of (33) becomes small when $\lambda$ approximates to 1 , since $u_{I}$ is in $\operatorname{cart}^{1}\left(I, \mathbf{R}^{N}\right)$.

By (22), (28), (31) and (33), we then obtain

$$
\int_{\operatorname{co}(F \cup \lambda F)}|M(D v)| d \mathcal{H}^{3} \leq 2 \sqrt{2} \delta \int_{F}\left|M\left(D u_{F}\right)\right| d \mathcal{H}^{2}+\eta+O(1-\lambda) .
$$

Repeating the argument for each 2-face $F$ of $Q_{(2)}$ (up to a rotation of $Q$ ), by (34) we finally infer

$$
\int_{\Sigma_{(3)}}|M(D v)| d \mathcal{H}^{3} \leq 2 \sqrt{2} \delta \sum_{F \in Q_{(2)}} \int_{F}\left|M\left(D u_{F}\right)\right| d \mathcal{H}^{2}+\varepsilon
$$

for any fixed small $\varepsilon>0$, taking $\lambda$ close to 1 and $\eta>0$ small.

We are now able to define $v$ on the 3-skeleton $Q_{(3)}$, first considering the easier case $n=3$.

Case $n=3$ : Since $Q=Q_{(3)}$ and $\Sigma_{(3)}=Q \backslash \operatorname{int}(\lambda Q)$, we set

$$
v(x)=u(x / \lambda) \quad \text { if } x \in \lambda Q .
$$

Since $Q_{(2)}=\partial Q$ and $v_{\mid F}=v_{F}$ for $F \in Q_{(2)}$, (22) yields (for $\eta>0$ small)

$$
\mathbf{M}\left(G_{v_{\mid \partial Q}}\left\llcorner\partial Q \times \mathbf{R}^{N}\right) \leq \sum_{F \in Q_{(2)}} \int_{F}\left|M\left(D u_{F}\right)\right| d \mathcal{H}^{2}+\varepsilon\right.
$$

whereas by (35), (36) and the area formula it follows that

$$
\begin{aligned}
\mathbf{M}\left(G_{v}\left\llcorner Q \times \mathbf{R}^{N}\right) \leq\right. & \mathbf{M}\left(G_{u}\left\llcorner Q \times \mathbf{R}^{N}\right)\right. \\
& +2 \sqrt{2} \delta \sum_{F \in Q_{(2)}} \int_{F}\left|M\left(D u_{F}\right)\right| d \mathcal{H}^{2}+\varepsilon .
\end{aligned}
$$


Unfortunately, $v$ is not continuous in $Q$ and, in case of codimension $N \geq 3$, in general $v$ is not in $\operatorname{cart}^{1}\left(Q, \mathbf{R}^{N}\right)$, since one has

$$
\partial G_{v}\left\llcorner\operatorname{int}(Q) \times \mathbf{R}^{N} \neq 0 .\right.
$$

More precisely, let $\llbracket \partial Q \rrbracket=\sum_{i, j=1}^{3,2}(-1)^{i+j+1} \llbracket F_{i}^{j} \rrbracket$, where $F_{i}^{j}, j=1,2$, are the 2-faces parallel to the plane $\left\{x \in \mathbf{R}^{3} \mid x_{i}=0\right\}$. Then, by defining

$$
p_{i}^{j}:=(-1)^{j} \delta \frac{\lambda+1}{2} e_{i}, \quad i=1,2,3, \quad j=1,2,
$$

where $\left\{e_{i}\right\}_{i=1}^{3}$ is the canonical basis of $\mathbf{R}^{3}$, one infers that $v$ is not continuous on the $p_{i}^{j}$, s and (taking account of Remark 4 in $2^{\text {nd }}$ step), if $\delta_{p}$ denotes the Dirac mass centered at $p$,

$$
\partial G_{v}\left\llcorner\operatorname{int}(Q) \times \mathbf{R}^{N}=\sum_{i, j=1}^{3,2}(-1)^{i+j} \delta_{p_{i}^{j}} \times\left(v_{F_{i}^{j} \#} \llbracket F_{i}^{j} \rrbracket-u_{F_{i}^{j} \#} \llbracket F_{i}^{j} \rrbracket\right) .\right.
$$

To overcome this fact, we make use of the remark ii) at the beginning of this section to "fill in" the holes of the graph. We then obtain in (41) an i.m. rectifiable current $T$ with boundary equal to the trace of $v$ on $\partial Q$, see (42), and mass control, see (43). Secondly, by Proposition 1 we are able to extend $v_{\mid \partial Q}$ to obtain a continuous Cartesian map $w$ with mapping area comparable to the mass of $T$, see (44). Finally, we change the parametrization of $w$ on $Q$ to obtain a control of the integral of the minors of smaller order of $D w$, see (45), (46) and (47).

Let now $R_{F_{i}^{j}} \in \mathcal{R}_{3}\left(\mathbf{R}^{N}\right)$ be the i.m. rectifiable current for which (23) and (24) hold w.r.t. $F=F_{i}^{j}$ and with $\eta=\varepsilon / 6$. Taking

$$
T:=G_{v}\left\llcorner Q \times \mathbf{R}^{N}-\sum_{i, j=1}^{3,2}(-1)^{i+j} \delta_{p_{i}^{j}} \times R_{F_{i}^{j}}\right.
$$

( $T=G_{v}\left\llcorner Q \times \mathbf{R}^{N}\right.$ in case $\left.N=2\right), T \in \mathcal{R}_{3}\left(\mathbf{R}^{n+N}\right)$ is such that by (40) $\partial T\left\llcorner\operatorname{int}(Q) \times \mathbf{R}^{N}=0\right.$ and therefore

$$
\partial T=G_{v_{\mid \partial Q}}\left\llcorner\partial Q \times \mathbf{R}^{N}=(I d \bowtie v)_{\mid \partial Q \# \llbracket \partial Q \rrbracket,}\right.
$$

with $v_{\mid \partial Q}: \partial Q \rightarrow \mathbf{R}^{N}$ continuous map in $\operatorname{cart}^{1}\left(\partial Q, \mathbf{R}^{N}\right)$ for which (37) holds, with restrictions $v_{\mid I}$ in $\operatorname{cart}^{1}\left(I, \mathbf{R}^{N}\right)$ for each 1 -face $I$ of $Q_{(1)}$, i.e., $\partial Q$ is in generic position w.r.t. $v_{\mid \partial Q}$. Moreover one has

$$
\begin{aligned}
\mathbf{M}(T) & =\mathbf{M}\left(G_{v}\left\llcorner Q \times \mathbf{R}^{N}\right)+\sum_{i, j=1}^{3,2} \mathbf{M}\left(R_{F_{i}^{j}}\right)\right. \\
& \leq \mathbf{M}\left(G_{v}\left\llcorner Q \times \mathbf{R}^{N}\right)+\varepsilon .\right.
\end{aligned}
$$


Then, by Proposition 1 (with $k=3$ ) there exists a map $w: Q \rightarrow \mathbf{R}^{N}$ in $\operatorname{cart}^{1}\left(Q, \mathbf{R}^{N}\right)$, continuous up to the closure and with boundary

$$
\partial\left(G_{w}\left\llcorner Q \times \mathbf{R}^{N}\right)=G_{v_{\mid \partial Q}}\left\llcorner\partial Q \times \mathbf{R}^{N},\right.\right.
$$

such that

$$
\int_{Q}\left|M_{(3)}(D w)\right| d x \leq \mathbf{M}(T)+\varepsilon
$$

if $N \geq 3,\left|M_{(3)}(D w)\right| \equiv 0$ if $N=2$.

Finally, to estimate the minors of smaller order of $D w$, for fixed $0<\sigma<1$, we define $v_{\varepsilon}: Q \rightarrow \mathbf{R}^{N}$ by

$$
v_{\varepsilon}(x):=\left\{\begin{array}{lll}
w(x / \sigma) & \text { if } & \|x\|_{3} \leq \sigma \delta \\
v\left(\delta x /\|x\|_{3}\right) & \text { if } & \sigma \delta \leq\|x\|_{3} \leq \delta
\end{array}\right.
$$

where $\|x\|_{3}:=\max _{1 \leq i \leq 3}\left|x_{i}\right|$. Then $v_{\varepsilon}$ is continuous in $Q$ and belongs to $\operatorname{cart}^{1}\left(Q, \mathbf{R}^{N}\right)$. In fact, by changing variable one has

$$
\int_{\left\{\|x\|_{3} \leq \sigma \delta\right\}}\left|M_{(j)}\left(D v_{\varepsilon}\right)\right| d x=\sigma^{3-j} \int_{Q}\left|M_{(j)}(D w)\right| d x
$$

for each $j=1, \ldots, 3$, so that for $\sigma$ sufficiently small

$$
\int_{\left\{\|x\|_{3} \leq \sigma \delta\right\}}\left|M\left(D v_{\varepsilon}\right)\right| d x \leq \int_{Q}\left|M_{(3)}(D w)\right| d x+\varepsilon
$$

( $\leq \varepsilon$ if $N=2$ ). Also, since $v_{\varepsilon}\left(\left\{\sigma \delta \leq\|x\|_{3} \leq \delta\right\}\right)$ is (at most) 2-dimensional one has

$$
\int_{\left\{\sigma \delta \leq\|x\|_{3} \leq \delta\right\}}\left|M_{(3)}\left(D v_{\varepsilon}\right)\right| d x=0
$$

whereas for $\mathcal{H}^{3}$-a.e. $\sigma \delta \leq\|x\|_{3} \leq \delta$ and for $j=1,2$ one has

$$
\left|M_{(j)}\left(D_{x} v_{\varepsilon}(x)\right)\right| \leq c\left(\frac{\delta}{\|x\|_{3}}\right)^{j}\left|M_{(j)}\left(D_{\tau} v(y)\right)\right|
$$

where $y=\delta x /\|x\|_{3}, \tau$ is an orthonormal basis for the boundary $\partial Q$ and $c>0$ is an absolute constant. Then, by the coarea formula and changing variable we infer

$$
\begin{aligned}
& \int_{\left\{\sigma \delta \leq\|x\|_{3} \leq \delta\right\}}\left|M\left(D v_{\varepsilon}\right)\right| d x \leq \\
\leq & c \int_{\sigma \delta}^{\delta} d \rho \int_{\left\{\|x\|_{3}=\rho\right\}} \sqrt{1+\left(\frac{\delta}{\rho}\right)^{2}\left|D_{\tau} v(y)\right|^{2}+\left(\frac{\delta}{\rho}\right)^{4}\left|M_{(2)}\left(D_{\tau} v(y)\right)\right|^{2}} d \mathcal{H}^{2} \\
\leq & c \int_{\sigma \delta}^{\delta} d \rho \int_{\left\{\|x\|_{3}=\delta\right\}} \sqrt{1+\left|D_{\tau} v\right|^{2}+\left|M_{(2)}\left(D_{\tau} v\right)\right|^{2}} d \mathcal{H}^{2} \\
= & c \delta(1-\sigma) \mathbf{M}\left(G_{v_{\mid \partial Q}}\left\llcorner\partial Q \times \mathbf{R}^{N}\right) .\right.
\end{aligned}
$$


By (37), (38), (43), (44), (45) and (47) we thus obtain

$$
\begin{aligned}
\mathbf{M}\left(G_{v_{\varepsilon}}\left\llcorner Q \times \mathbf{R}^{N}\right)\right. & \leq \mathbf{M}\left(G_{u}\left\llcorner Q \times \mathbf{R}^{N}\right)\right. \\
& +(2 \sqrt{2}+c) \delta \sum_{F \in Q_{(2)}} \int_{F}\left|M\left(D u_{F}\right)\right| d \mathcal{H}^{2}+(4+c \delta) \varepsilon
\end{aligned}
$$

as required in (25).

Case $n>3$ : To extend $v$ to the 3-skeleton $Q_{(3)}$, we adapt the argument of the case of dimension $n=3$. To this aim, let $K$ be a 3-face of $Q_{(3)}$, and choose for simplicity

$$
K=\left\{x \in \mathbf{R}^{n} \mid x=(\tilde{x}, \widehat{\delta}),\|\widetilde{x}\|_{3} \leq \delta\right\},
$$

where now $\tilde{x}:=\left(x_{1}, x_{2}, x_{3}\right) \in \mathbf{R}^{3}, \widehat{t}:=(t, \ldots, t) \in \mathbf{R}^{n-3},\|\widetilde{x}\|_{3}:=\max _{1 \leq i \leq 3}\left|x_{i}\right|$. We have $\llbracket \partial K \rrbracket=\sum_{i, j=1}^{3,2}(-1)^{i+j+1} \llbracket F_{i}^{j} \rrbracket$, where $F_{i}^{j}, j=1,2$, are the 2-faces parallel to the plane $\left\{x \in \mathbf{R}^{n} \mid x_{h}=0\right.$ if $\left.h=i, 4, \ldots, n\right\}$, for $1 \leq i \leq 3$. Since the map $v$ is already given on the subset of $\Sigma_{(3)}$

$$
\bigcup_{i, j=1}^{3,2} \operatorname{co}\left(F_{i}^{j} \cup \lambda F_{i}^{j}\right)=\left\{(t \tilde{x}, t \widehat{\delta}) \mid \lambda \leq t \leq 1, \quad\|\tilde{x}\|_{3}=\delta\right\}
$$

we define $v$ on $K$ by

$$
v(\widetilde{x}, \widehat{\delta})=u(\widetilde{x} / \lambda, \widehat{\delta}) \quad \text { if } \quad\|\widetilde{x}\|_{3} \leq \delta \lambda
$$

whereas on $K \bigcap\left\{(t \tilde{x}, \widehat{\delta}) \mid \lambda \leq t \leq 1,\|\tilde{x}\|_{3}=\delta\right\}$ we take $v$ as it is given in $\operatorname{co}\left(F_{i}^{j} \cup \lambda F_{i}^{j}\right)$, i.e.,

$$
v(t \tilde{x}, \widehat{\delta})=v(t \tilde{x}, t \widehat{\delta}) \quad \text { if } \quad \lambda \leq t \leq 1, \quad\|\widetilde{x}\|_{3}=\delta
$$

Then, by a computation similar to the case $n=3$, the mass estimates (37) and (38) hold again (with $K$ instead of $Q$ and $F \in Q_{(2)}, F \subset \partial K$ ). Anyway, in general $v$ is neither continuous nor a Cartesian map. More precisely, if $p_{i}^{j}$ is defined by (39), where $\left\{e_{i}\right\}_{i=1}^{n}$ is the canonical basis of $\mathbf{R}^{n}, v_{\mid K}$ is not continuous on the $p_{i}^{j}$,s and (40) holds (with $K$ for $Q$ ). Then, taking $T$ as in (41) $\left(T=G_{v}\left\llcorner K \times \mathbf{R}^{N}\right.\right.$ if $N=2$ ), where $R_{F_{i}^{j}} \in \mathcal{R}_{3}\left(\mathbf{R}^{N}\right)$ is given by (23) and (24), (42) holds again. Applying Proposition 1 with $Q=K$, working similarly to the case $n=3$ to estimate the minors of smaller order, and repeating the argument for each $K \in Q_{(3)}$, we finally define a continuous map $v: Q_{(3)} \rightarrow \mathbf{R}^{N}$ such that $v_{K}$ is in $\operatorname{cart}^{1}\left(K, \mathbf{R}^{N}\right)$ and

$$
\begin{aligned}
\int_{K}\left|M\left(D v_{K}\right)\right| d \mathcal{H}^{3} \leq & \int_{K}\left|M\left(D u_{K}\right)\right| d \mathcal{H}^{3} \\
& +c \delta \sum_{\substack{F \in Q_{(2)} \\
F \subset \partial K}} \int_{F}\left|M\left(D u_{F}\right)\right| d \mathcal{H}^{2}+\eta
\end{aligned}
$$

for each 3-face $K$ of $Q_{(3)}$, where $v_{K}:=v_{\mid K}$ and $u_{K}:=u_{\mid K}$. 
Remark 5. Note that if $K_{1}, K_{2} \in Q_{(3)}$ are such that $\partial K_{1} \cap \partial K_{2}=F \in Q_{(2)}$, then one has

$$
\partial\left(G _ { v _ { | K _ { i } } } \llcorner \operatorname { i n t } ( K _ { i } ) \times \mathbf { R } ^ { N } ) \left\llcornerF \times \mathbf{R}^{N}=\sigma_{i} G_{v_{\mid F}}\left\llcorner F \times \mathbf{R}^{N}\right.\right.\right.
$$

for $i=1,2$, where $\sigma_{1}=-\sigma_{2}= \pm 1$, according to the orientations.

In case $n>3$, and for each $k=4, \ldots, n$, by an adaptation of $3^{r d}$ step we have the following

$k^{\text {th }}$ step: definition of $v$ on $\Sigma_{(k)} \cup Q_{(k)}$.

At the $(k-1)^{t h}$ step, $v$ has been defined on $\Sigma_{(k-1)}$ so that for each $(k-2)$-face $F$ of $Q_{(k-2)}$

$$
\begin{array}{r}
\int_{\operatorname{co}(F \cup \lambda F)}|M(D v)| d \mathcal{H}^{k-1} \leq 2 \sqrt{k-2} \delta \int_{F}\left|M\left(D u_{F}\right)\right| d \mathcal{H}^{k-2}+\eta+O(1-\lambda) \\
+c(n, k-1) \sum_{j=1}^{k-4} \delta^{j+1} \sum_{\substack{I \in Q_{(k-2-j)} \\
I \subset \partial F}} \int_{I}\left|M\left(D u_{I}\right)\right| d \mathcal{H}^{k-2-j}
\end{array}
$$

(see (34) for $k=4)$, where $c(n, k-1)>0$ is an absolute constant, $\eta>0$ is small and $u_{F}:=u_{\mid F}, u_{I}:=u_{\mid I}$. Moreover, $v$ has been defined on $Q_{(k-1)}$ so that, for each $(k-1)$-face $K$ of $Q_{(k-1)}$ one has

$$
\begin{aligned}
\int_{K}\left|M\left(D v_{K}\right)\right| d \mathcal{H}^{k-1} & \leq \int_{K}\left|M\left(D u_{K}\right)\right| d \mathcal{H}^{k-1}+\eta \\
& +c \sum_{j=1}^{k-3} \delta^{j} \sum_{\substack{I \in Q_{(k-1-j)} \\
I \subset \partial K}} \int_{I}\left|M\left(D u_{I}\right)\right| d \mathcal{H}^{k-1-j}
\end{aligned}
$$

(see $(48)$ for $k=4$ ), where $c>0$ is an absolute constant and $v_{K}:=v_{\mid K}$, $u_{K}:=u_{\mid K}$.

Remark 6. Finally, Remark 5 at the end of $3^{r d}$ step holds again with $K_{i} \in Q_{(k-1)}$ and $F \in Q_{(k-2)}$.

To define $v$ on $\Sigma_{(k)}$, let us fix a $(k-1)$-face $K$ of $Q_{(k-1)}$, for simplicity

$$
K=\left\{x \in \mathbf{R}^{n} \mid x=(\widetilde{x}, \widehat{\delta}),\|\widetilde{x}\|_{k-1} \leq \delta\right\}
$$

where now $\tilde{x}:=\left(x_{1}, \ldots, x_{k-1}\right) \in \mathbf{R}^{k-1}, \widehat{t}:=(t, \ldots, t) \in \mathbf{R}^{n-k+1},\|\tilde{x}\|_{k-1}$ $:=\max _{1 \leq i \leq k-1}\left|x_{i}\right|$. By an argument similar to the one in the first part of $3^{r d}$ step, we will define $v$ on the convex hull

$$
\operatorname{co}(K \cup \lambda K)=\left\{(\widetilde{x}, \widehat{t}) \mid \lambda \delta \leq t \leq \delta, \quad\|\widetilde{x}\|_{k-1} \leq t\right\}
$$

by using the maps $u_{K}, v_{K}$ and the trace of $v$ on $\Sigma_{(k-1)}$. We then divide in three parts

$$
\operatorname{co}(K \cup \lambda K)=\bigcup_{i=1}^{3} \Delta_{K}^{i},
$$


where the $\Delta_{K}^{i}$ 's are given by (26), (29) and (32), respectively, with $\tilde{x} \in \mathbf{R}^{k-1}$, $\widehat{t} \in \mathbf{R}^{n-k+1}$ and $\|\cdot\|_{k-1}$ instead of $\|\cdot\|_{2}$.

Using the trace $v_{K}$ of $v$ on $K$ given at the $(k-1)^{\text {th }}$ step, see (50), define $v$ on $\Delta_{K}^{1}$ by means of the implicit map

$$
\begin{gathered}
\left.\left.G^{(1)}:\right] \delta\left(\frac{\lambda+1}{2}\right), \delta\right] \times[-\delta, \delta]^{k-1} \longrightarrow \Delta_{K}^{1} \times \mathbf{R}^{N} \\
G^{(1)}(t, \tilde{x}):=\left((f(t) \tilde{x}, \widehat{t}), v_{K}(\tilde{x}, \widehat{\delta})\right)
\end{gathered}
$$

with $f$ given by (27). We then infer

$$
\int_{\Delta_{K}^{1}}|M(D v)| d \mathcal{H}^{k} \leq \sqrt{k-1} \delta \int_{K}\left|M\left(D v_{K}\right)\right| d \mathcal{H}^{k-1}+O(1-\lambda) .
$$

In fact, as remarked at the beginning of $3^{r d}$ step, by the area formula one has

$$
\int_{\Delta_{K}^{1}}|M(D v)| d \mathcal{H}^{k}=\int_{\delta\left(\frac{\lambda+1}{2}\right)}^{\delta} d t \int_{[-\delta, \delta]^{k-1}} J_{k} G^{(1)} d \mathcal{H}^{k-1}
$$

where $J_{k} G^{(1)}$ is the $k$-dimensional Jacobian of $G^{(1)}$. By direct computation

$$
\begin{aligned}
& \left(J_{k} G^{(1)}\right)^{2} \leq(n-k+1) f(t)^{2(k-1)} \\
& \quad+\sum_{j=1}^{k-1} f(t)^{2(k-1-j)}\left[(n-k+1)+\left(\frac{2}{\delta(1-\lambda)}\right)^{2}|\widetilde{x}|^{2}\right] \cdot\left|M_{(j)}\left(D v_{K}\right)\right|^{2}
\end{aligned}
$$

where $\left|M_{(j)}\left(D v_{K}\right)\right|=0$ if $j>N$ and $|\widetilde{x}|^{2}=\left(x_{1}\right)^{2}+\cdots+\left(x_{k-1}\right)^{2}$. Then, for $\lambda$ close to 1 we obtain (51).

Using the trace $u_{K}$ of the given map $u$ on $K$, let us define $v$ on $\Delta_{K}^{2}$ by means of the implicit map

$$
\begin{gathered}
G^{(2)}:\left[\delta \lambda, \delta\left(\frac{\lambda+1}{2}\right)\left[\times[-\delta, \delta]^{k-1} \longrightarrow \Delta_{K}^{2} \times \mathbf{R}^{N}\right.\right. \\
G^{(2)}(t, \tilde{x}):=\left((f(t) \tilde{x}, \widehat{t}), u_{K}(\tilde{x}, \widehat{\delta})\right)
\end{gathered}
$$

with $f$ given by (30). One then similarly obtains

$$
\int_{\Delta_{K}^{2}}|M(D v)| d \mathcal{H}^{k} \leq \sqrt{k-1} \delta \int_{K}\left|M\left(D u_{K}\right)\right| d \mathcal{H}^{k-1}+O(1-\lambda) .
$$

Finally, let us define $v$ on $\Delta_{K}^{3}$ by means of the trace of $v$ given at the $(k-1)^{t h}$ step on the subset of $\Sigma_{(k-1)}$

$$
\bigcup_{i=1}^{2(k-1)} \operatorname{co}\left(F_{i} \cup \lambda F_{i}\right)=\left\{(t \tilde{x}, t \widehat{\delta}) \mid \lambda \leq t \leq 1, \quad\|\tilde{x}\|_{k-1}=\delta\right\}
$$


where $F_{i} \in Q_{(k-2)}$ are such that $\partial K=\bigcup_{i=1}^{2(k-1)} F_{i}$. For fixed $(\widetilde{x}, \widehat{\delta}) \in \partial K$, so that $\|\widetilde{x}\|_{k-1}=\delta$, and for each $\left.\left.\sigma \in\right] 0,1\right], v$ describes with $t \in[\lambda, 1]$, on the parallel line segments connecting the points $\left(\lambda \sigma \widetilde{x}, \frac{1}{2}[(1+\lambda)+\sigma(\lambda-1)] \widehat{\delta}\right)$ and $\left(\sigma \widetilde{x}, \frac{1}{2}[(1+\lambda)+\sigma(1-\lambda)] \widehat{\delta}\right)$, the trace of $v$ given at the $(k-1)^{t h}$ step on the line segments connecting $(\lambda \widetilde{x}, \lambda \widehat{\delta})$ with $(\widetilde{x}, \widehat{\delta})$. More precisely, $v$ is defined on $\Delta_{K}^{3}$ by the implicit map

$$
\begin{gathered}
\left.\left.G^{(3)}:\left\{\tilde{x} \in \mathbf{R}^{k-1} \mid\|\tilde{x}\|_{k-1}=\delta\right\} \times[\lambda, 1] \times\right] 0,1\right] \longrightarrow \Delta_{K}^{3} \times \mathbf{R}^{N} \\
G^{(3)}(\tilde{x}, t, \sigma):=\left(\left(t \sigma \widetilde{x}, \frac{1}{2}[(1+\lambda)+\sigma(2 t-(1+\lambda))] \widehat{\delta}\right), v(t \tilde{x}, t \widehat{\delta})\right) .
\end{gathered}
$$

One then infers

$$
\begin{aligned}
& \int_{\Delta_{K}^{3}}|M(D v)| d \mathcal{H}^{k} \leq \\
& \leq c \sum_{j=1}^{k-3} \delta^{j+1} \sum_{\substack{I \in Q_{(k-1-j)} \\
I \subset \partial K}} \int_{I}\left|M\left(D u_{I}\right)\right| d \mathcal{H}^{k-1-j}+\eta+O(1-\lambda)
\end{aligned}
$$

for some absolute constant $c>0$. In fact, for example, on the subset

$$
\Delta_{K}^{3} \cap\left\{(\widetilde{x}, \widehat{t})|\delta \lambda \leq t \leq \delta,| x_{i} \mid \leq x_{1} \quad \forall i=2, \ldots, k-1\right\}
$$

corresponding to the $(k-2)$-face $F$ of $\partial K$

$$
F=\left\{\left(\delta, x_{2}, \ldots, x_{k-1}, \widehat{\delta}\right)|| x_{i} \mid \leq \delta \quad \forall i=2, \ldots, k-1\right\},
$$

the area of the graph of $v$ is smaller than the area of $v$ on $\operatorname{co}(F \cup \lambda F)$ times the length of its projection onto the orthogonal section to $\operatorname{co}(F \cup \lambda F)$, i.e., smaller than

$$
\delta \int_{\operatorname{co}(F \cup \lambda F)}|M(D v)| d \mathcal{H}^{k-1} .
$$

Then one has

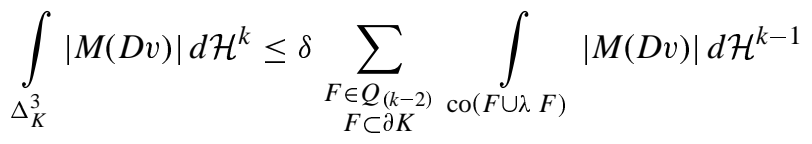

and by (49) one obtains (53). By (50), (51), (52) and (53) one then infers

$$
\begin{aligned}
\int_{\operatorname{co}(K \cup \lambda K)}|M(D v)| d \mathcal{H}^{k} \leq & 2 \sqrt{k-1} \delta \int_{K}\left|M\left(D u_{K}\right)\right| d \mathcal{H}^{k-1}+\eta+O(1-\lambda) \\
& +c(n, k) \sum_{j=1}^{k-3} \delta^{j+1} \sum_{\substack{I \in Q_{(k-1-j)} \\
I \subset \partial K}} \int_{I}\left|M\left(D u_{I}\right)\right| d \mathcal{H}^{k-1-j}
\end{aligned}
$$


(compare (49)). Repeating the argument for each $(k-1)$-face $K$ of $Q_{(k-1)}$, we finally obtain

$$
\begin{array}{r}
\int_{\Sigma_{(k)}}|M(D v)| d \mathcal{H}^{k} \leq 2 \sqrt{k-1} \delta \sum_{K \in Q_{(k-1)}} \int_{K}\left|M\left(D u_{K}\right)\right| d \mathcal{H}^{k-1}+\varepsilon \\
+\widetilde{c}(n, k) \sum_{j=1}^{k-3} \delta^{j+1} \sum_{I \in Q_{(k-1-j)}} \int_{I}\left|M\left(D u_{I}\right)\right| d \mathcal{H}^{k-1-j}
\end{array}
$$

for $\lambda$ close to 1 and $\eta>0$ small, where $c(n, k), \widetilde{c}(n, k)$ are absolute constant and $\varepsilon>0$ is small.

We are now able to define $v$ on the k-skeleton $Q_{(k)}$, first considering the easier case $n=k$, which is an adaptation of the case $n=3$ in $3^{r d}$ step.

Case $n=k$ : Since $Q=Q_{(n)}$ and $\Sigma_{(n)}=Q \backslash \operatorname{int}(\lambda Q)$, we set

$$
v(x)=u(x / \lambda) \quad \text { if } x \in \lambda Q .
$$

Since $Q_{(n-1)}=\partial Q,(50)$ yields (for $\eta>0$ small)

$$
\begin{aligned}
\mathbf{M}\left(G_{v_{\mid \partial Q}} L \partial Q \times \mathbf{R}^{N}\right) & \leq \sum_{K \in Q_{(n-1)}} \int_{K}\left|M\left(D u_{K}\right)\right| d \mathcal{H}^{n-1}+\varepsilon \\
& +c \sum_{j=1}^{n-3} \delta^{j} \sum_{I \in Q_{(n-1-j)}} \int_{I}\left|M\left(D u_{I}\right)\right| d \mathcal{H}^{n-1-j}
\end{aligned}
$$

whereas by (54), (55) and the area formula it follows that

$$
\begin{aligned}
\mathbf{M}\left(G_{v}\left\llcorner Q \times \mathbf{R}^{N}\right)\right. & \leq \mathbf{M}\left(G_{u}\left\llcorner Q \times \mathbf{R}^{N}\right)\right. \\
& +c \sum_{j=1}^{n-2} \delta^{j} \sum_{I \in Q_{(n-j)}} \int_{I}\left|M\left(D u_{I}\right)\right| d \mathcal{H}^{n-j}+\varepsilon .
\end{aligned}
$$

However $v$ is not continuous on $Q$ and, in case $N \geq 3$, in general

$$
\partial G_{v}\left\llcorner\operatorname{int}(Q) \times \mathbf{R}^{N} \neq 0 .\right.
$$

In fact, if $F$ is a 2-face of $Q_{(2)}$, for example

$$
F=\left\{\left(x_{1}, x_{2}, \delta, \ldots, \delta\right) \in \mathbf{R}^{n} \mid \max \left\{\left|x_{1}\right|,\left|x_{2}\right|\right\} \leq \delta\right\},
$$

then one can check that $v$ is not continuous on the $(n-3)$-dimensional subset $S_{F}$ of the boundary of the $(n-2)$-cube

$$
\left\{x \in \mathbf{R}^{n} \mid x_{1}=x_{2}=0, \quad 0 \leq x_{i} \leq \delta \frac{\lambda+1}{2} \quad \forall i=3, \ldots, n\right\}
$$

given by

$$
S_{F}:=\left\{x \mid x_{1}=x_{2}=0, \quad x_{i} \geq 0 \quad \forall i=3, \ldots, n, \quad \max _{3 \leq i \leq n} x_{i}=\delta \frac{\lambda+1}{2}\right\} .
$$


Also, if the i.m. rectifiable current $\llbracket S_{F} \rrbracket \in \mathcal{R}_{n-3}\left(\mathbf{R}^{n}\right)$ is equipped with the natural orientation induced by such $(n-2)$-cube onto its boundary, and $Q_{F}$ is the subset of $Q$ given by

$$
Q_{F}:=\left\{x \in Q \mid \min \left\{x_{3}, \ldots, x_{n}\right\} \geq \max \left\{\left|x_{1}\right|,\left|x_{2}\right|\right\}\right\}
$$

then one has

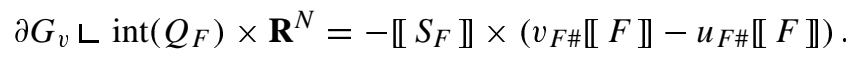

Finally, taking account of formula $[16,26.17]$ for the boundary of cartesian products, we compute

$$
\begin{aligned}
& \partial\left(G_{v}\left\llcorner\operatorname{int}\left(Q_{F}\right) \times \mathbf{R}^{N}\right)=\right. \\
& \quad=G_{v_{\mid \partial Q_{F}}}\left\llcorner\partial Q_{F} \times \mathbf{R}^{N}-(-1)^{n-3} \sigma_{F} \partial\left(\llbracket S_{F} \rrbracket \times R_{F}\right)\right.
\end{aligned}
$$

where $\sigma_{F}=1$ and $R_{F} \in \mathcal{R}_{3}\left(\mathbf{R}^{N}\right)$ is the i.m. rectifiable current for which (23) and (24) hold. Similarly, if $Q_{F_{i}} \subset Q, \llbracket S_{F_{i}} \rrbracket \in \mathcal{R}_{n-3}\left(\mathbf{R}^{n}\right)$ and $R_{F_{i}} \in \mathcal{R}_{3}\left(\mathbf{R}^{N}\right)$ are defined in analogous way w.r.t. each 2-face $F_{i}$ of $Q_{(2)}$ (up to a rotation of $Q$ so that $F_{i}$ coincides with $F$ ), we obtain (58) with $F=F_{i}$ and suitable $\sigma_{F_{i}}= \pm 1$, according to orientations. Since the $Q_{F_{i}}$ 's are pairwise interiorly disjoint and cover all the $n$-cube $Q$, defining $T \in \mathcal{R}_{n}\left(\mathbf{R}^{n+N}\right)$ by

$$
T=G_{v}\left\llcorner Q \times \mathbf{R}^{N}+(-1)^{n-3} \sum_{F_{i} \in Q_{(2)}} \sigma_{F_{i}} \llbracket S_{F_{i}} \rrbracket \times R_{F_{i}}\right.
$$

( $T=G_{v}\left\llcorner Q \times \mathbf{R}^{N}\right.$ if $N=2$ ), taking account of Remark 6 at the beginning of $k^{\text {th }}$ step, we obtain $\partial T\left\llcorner\operatorname{int}(Q) \times \mathbf{R}^{N}=0\right.$. We therefore infer

$$
\partial T=G_{v_{\mid \partial Q}}\left\llcorner\partial Q \times \mathbf{R}^{N}=(I d \bowtie v)_{\mid \partial Q \# \llbracket \partial Q \rrbracket,}\right.
$$

where $v_{\mid \partial Q}: \partial Q \rightarrow \mathbf{R}^{N}$ is a continuous map in $\operatorname{cart}^{1}\left(\partial Q, \mathbf{R}^{N}\right)$ for which (56) holds. Also, $\partial Q$ is in generic position w.r.t. $v_{\mid \partial Q}$, since the restriction $v_{\mid I}$ is in $\operatorname{cart}^{1}\left(I, \mathbf{R}^{N}\right)$ for each $(n-j)$-face $I$ of $Q_{(n-j)}, j=2, \ldots, n-1$. Moreover, since $\mathcal{H}^{n-3}\left(S_{F_{i}}\right)=c(n)\left[\delta\left(\frac{\lambda+1}{2}\right)\right]^{n-3}$ and the masses $\mathbf{M}\left(R_{F_{i}}\right)$ are taken arbitrary small one has

$$
\mathbf{M}(T) \leq \mathbf{M}\left(G_{v}\left\llcorner Q \times \mathbf{R}^{N}\right)+c(n) \delta^{n-3} \varepsilon .\right.
$$

Then, by Proposition 1 (with $k=n$ ) there exists a map $w: Q \rightarrow \mathbf{R}^{N}$ in $\operatorname{cart}^{1}\left(Q, \mathbf{R}^{N}\right)$, continuous up to the closure of $Q$ and with boundary

$$
\partial\left(G_{w}\left\llcorner Q \times \mathbf{R}^{N}\right)=G_{v_{\mid \partial Q}}\left\llcorner\partial Q \times \mathbf{R}^{N},\right.\right.
$$

such that

$$
\int_{Q}\left|M_{(n)}(D w)\right| d x \leq \mathbf{M}(T)+\varepsilon
$$

if $N \geq n,\left|M_{(n)}(D w)\right| \equiv 0$ if $N<n$. 
Again, to estimate the minors of smaller order of $D w$, for fixed $0<\sigma<1$, we define $v_{\varepsilon}: Q \rightarrow \mathbf{R}^{N}$ by

$$
v_{\varepsilon}(x):=\left\{\begin{array}{lll}
w(x / \sigma) & \text { if } & \|x\|_{n} \leq \sigma \delta \\
v\left(\delta x /\|x\|_{n}\right) & \text { if } & \sigma \delta \leq\|x\|_{n} \leq \delta
\end{array}\right.
$$

where $\|x\|_{n}:=\max _{1 \leq i \leq n}\left|x_{i}\right|$. Then $v_{\varepsilon}$ is continuous on $Q$ and belongs to $\operatorname{cart}^{1}\left(Q, \mathbf{R}^{N}\right)$. In fact, similarly to the case $n=3$ of $3^{r d}$ step, for each $j=1, \ldots, n$ one has

$$
\int_{\left\{\|x\|_{n} \leq \sigma \delta\right\}}\left|M_{(j)}\left(D v_{\varepsilon}\right)\right| d x=\sigma^{n-j} \int_{Q}\left|M_{(j)}(D w)\right| d x
$$

so that for $\sigma$ sufficiently small

$$
\int_{\left\{\|x\|_{n} \leq \sigma \delta\right\}}\left|M\left(D v_{\varepsilon}\right)\right| d x \leq \int_{Q}\left|M_{(n)}(D w)\right| d x+\varepsilon
$$

$(\leq \varepsilon$ if $N<n)$ whereas, since

$$
\int_{\left\{\sigma \delta \leq\|x\|_{n} \leq \delta\right\}}\left|M_{(n)}\left(D v_{\varepsilon}\right)\right| d x=0
$$

and (46) holds again for each $j=1, \ldots, n-1$ and for $\mathcal{H}^{n}$-a.e. $x$, with $\|\cdot\|_{n}$ instead of $\|\cdot\|_{3}$, similarly to (47) one infers

$$
\int_{\left\{\sigma \delta \leq\|x\|_{n} \leq \delta\right\}}\left|M\left(D v_{\varepsilon}\right)\right| d x \leq c \delta \mathbf{M}\left(G_{v_{\mid \partial Q}}\left\llcorner\partial Q \times \mathbf{R}^{N}\right) .\right.
$$

Therefore, by (56), (57), (59), (60), (61) and (62) we obtain (as $\delta<1$ )

$$
\begin{aligned}
\mathbf{M}\left(G_{v_{\varepsilon}}\left\llcorner Q \times \mathbf{R}^{N}\right)\right. & \leq \mathbf{M}\left(G_{u}\left\llcorner Q \times \mathbf{R}^{N}\right)+c_{1} \varepsilon\right. \\
& +c_{2} \sum_{j=1}^{n-2} \delta^{j} \sum_{I \in Q_{(n-j)}} \int_{I}\left|M\left(D u_{I}\right)\right| d \mathcal{H}^{n-j}
\end{aligned}
$$

for some absolute constants $c_{1}, c_{2}>0$, as required in (25).

Case $n>k$ : To define $v$ on $Q_{(k)}$, we adapt the argument of the case $n=k$. To this aim, let $L$ be a $k$-face of $Q_{(k)}$, and choose for simplicity

$$
L=\left\{x \in \mathbf{R}^{n} \mid x=(\tilde{x}, \widehat{\delta}),\|\tilde{x}\|_{k} \leq \delta\right\},
$$

where now $\tilde{x}:=\left(x_{1}, \ldots, x_{k}\right) \in \mathbf{R}^{k}, \widehat{t}:=(t, \ldots, t) \in \mathbf{R}^{n-k},\|\widetilde{x}\|_{k}:=\max _{1 \leq i \leq k}\left|x_{i}\right|$. If $\partial L=\bigcup_{j=1}^{2 k} K_{j}$, with $K_{j} \in Q_{(k-1)}$, the map $v$ is already defined on the subset of $\Sigma_{(k)}$

$$
\bigcup_{j=1}^{2 k} \operatorname{co}\left(K_{j} \cup \lambda K_{j}\right)=\left\{(t \tilde{x}, t \widehat{\delta}) \mid \lambda \leq t \leq 1, \quad\|\tilde{x}\|_{k}=\delta\right\} .
$$


Define now $v$ on $L$ by

$$
v(\widetilde{x}, \widehat{\delta})=u(\tilde{x} / \lambda, \widehat{\delta}) \quad \text { if } \quad\|\widetilde{x}\|_{k} \leq \delta \lambda
$$

whereas on $L \bigcap\left\{(t \tilde{x}, \widehat{\delta}) \mid \lambda \leq t \leq 1,\|\widetilde{x}\|_{k}=\delta\right\}$ we take $v$ as it is given in the $\operatorname{co}\left(K_{j} \cup \lambda K_{j}\right)$ 's, i.e., we set

$$
v(t \tilde{x}, \widehat{\delta})=v(t \tilde{x}, t \widehat{\delta}) \quad \text { if } \quad \lambda \leq t \leq 1, \quad\|\widetilde{x}\|_{k}=\delta
$$

Then, by a computation similar to the case $n=k$, the mass estimates (56) and (57) hold again (with $L$ and $k$ instead of $Q$ and $n$, respectively, $K \subset \partial L$ and $I \subset \partial L$ ). Moreover, $v$ is not continuous on $K$ and (for $N \geq 3$ ) in general

$$
\partial G_{v}\left\llcorner\operatorname{int}(K) \times \mathbf{R}^{N} \neq 0 .\right.
$$

In fact, if $F \in Q_{(2)}$ is a 2-face of the boundary of $L, F \subset \partial L$, for example

$$
F=\left\{\left(x_{1}, x_{2}, \delta, \ldots, \delta\right) \in \mathbf{R}^{n} \mid \max \left\{\left|x_{1}\right|,\left|x_{2}\right|\right\} \leq \delta\right\},
$$

$v$ is not continuous on the $(k-3)$-dimensional subset $\widetilde{S}_{F}$ (depending on $L$ ) of the boundary of the $(k-2)$-cube

$$
\left\{(\tilde{x}, \widehat{\delta}) \mid x_{1}=x_{2}=0, \quad 0 \leq x_{i} \leq \delta \frac{\lambda+1}{2} \quad \forall i=3, \ldots, k\right\}
$$

given by

$$
\widetilde{S}_{F}:=\left\{(\widetilde{x}, \widehat{\delta}) \mid x_{1}=x_{2}=0, x_{i} \geq 0 \forall i=3, \ldots, k, \max _{3 \leq i \leq k} x_{i}=\delta \frac{\lambda+1}{2}\right\} .
$$

Also, if $\llbracket \widetilde{S}_{F} \rrbracket \in \mathcal{R}_{k-3}\left(\mathbf{R}^{n}\right)$ is equipped with the natural orientation induced by such $(k-2)$-cube onto its boundary, and $\widetilde{Q}_{F}$ is the corresponding subset of $L$ given by

$$
\widetilde{Q}_{F}:=\left\{(\widetilde{x}, \widehat{\delta}) \in \mathbf{R}^{n} \mid \min \left\{x_{3}, \ldots, x_{k}\right\} \geq \max \left\{\left|x_{1}\right|,\left|x_{2}\right|\right\}\right\},
$$

we infer that (58) holds again, with $k, \widetilde{Q}_{F}$ and $\widetilde{S}_{F}$ instead of $n, Q_{F}$ and $S_{F}$, respectively, and $R_{F} \in \mathcal{R}_{3}\left(\mathbf{R}^{N}\right)$ given by (23) and (24). If $\widetilde{Q}_{F_{i}} \subset Q$, 【 $\widetilde{S}_{F_{i}} \rrbracket \in$ $\mathcal{R}_{k-3}\left(\mathbf{R}^{n}\right)$ and $R_{F_{i}} \in \mathcal{R}_{3}\left(\mathbf{R}^{N}\right)$ are defined in analogous way for each 2-face $F_{i}$ of $Q_{(2)}$ contained in $\partial L$, for suitable $\sigma_{F_{i}}= \pm 1$, we take

$$
T=G_{v}\left\llcorner L \times \mathbf{R}^{N}+(-1)^{k-3} \sum_{\substack{F_{i} \in Q_{(2)} \\ F_{i} \subset \partial L}} \sigma_{F_{i}} \llbracket \widetilde{S}_{F_{i}} \rrbracket \times R_{F_{i}} \in \mathcal{R}_{k}\left(\mathbf{R}^{n+N}\right)\right.
$$

$\left(T=G_{v}\left\llcorner L \times \mathbf{R}^{N}\right.\right.$ if $\left.N=2\right)$, so that $\partial T\left\llcorner\operatorname{int}(L) \times \mathbf{R}^{N}=0\right.$ and

$$
\partial T=G_{v_{\mid \partial L}}\left\llcorner\partial L \times \mathbf{R}^{N}=(I d \bowtie v)_{\mid \partial L \# \llbracket \partial L \rrbracket .}\right.
$$


Applying Proposition 1 with $Q=L$, working similarly to the case $n=k$ and repeating the argument for each $L \in Q_{(k)}$, we then define a continuous map $v: Q_{(k)} \rightarrow \mathbf{R}^{N}$ such that $v_{L}$ is in $\operatorname{cart}^{1}\left(L, \mathbf{R}^{N}\right)$ with

$$
\begin{aligned}
\int_{L}\left|M\left(D v_{L}\right)\right| d \mathcal{H}^{k} & \leq \int_{L}\left|M\left(D u_{L}\right)\right| d \mathcal{H}^{k}+\eta \\
& +c \sum_{j=1}^{k-2} \delta^{j} \sum_{\substack{I \in Q_{(k-j)} \\
I \subset \partial L}} \int_{I}\left|M\left(D u_{I}\right)\right| d \mathcal{H}^{k-j}
\end{aligned}
$$

for each $k$-face $L \in Q_{(k)}$, where $v_{L}:=v_{\mid L}, u_{L}:=u_{\mid L}$ and $c>0$ is an absolute constant, compare (50). Finally, Remark 6 holds again with $K_{i} \in Q_{(k)}$ and $F \in Q_{(k-1)}$, as required.

\section{Partition of a bounded open set}

In this section we define a suitable partition of an open subset $U$ of $\Omega, U \subset \subset \Omega$, $\Omega \subset \mathbf{R}^{n}$ being the domain of a map $u$ in $\operatorname{cart}^{1}\left(\Omega, \mathbf{R}^{N}\right)$. Each polyhedral region $Q$ of the partition is chosen in such a way that $Q$ is in generic position w.r.t. $u$, compare Definition 2 in Sect. 1. Also, nice integral estimates hold for the restriction of $u$ to the skeleton of $Q$, see (63). To this aim we first recall the notion of Whitney family of cubes $\operatorname{WF}(U)$ associated to the bounded open set $U \subset \mathbf{R}^{n}$, compare [2] or [13].

Denote by $\mathbf{K}(0)$ the family of closed unit $n$-dimensional cubes associated to the integer grid $\mathbf{Z}^{n}$ of $\mathbf{R}^{n}$. For each integer $l \in \mathbf{Z}$, denote by $\mathbf{K}(l)$ the family of standard cubes

$$
\mathbf{K}(l):=\left\{2^{-l} K \mid K \in \mathbf{K}(0)\right\} .
$$

Cubes of $\mathbf{K}(l)$ are said of level $l$ and we set level $(K)=l$ if $K \in \mathbf{K}(l)$. Finally set

$$
\mathbf{K}:=\bigcup_{l \in \mathbf{Z}} \mathbf{K}(l)
$$

Consider the edge metric

$$
\delta(x, y):=\sup \left\{\left|x_{i}-y_{i}\right| \mid i \in\{1, \ldots, n\}\right\}
$$

for $x, y \in \mathbf{R}^{n}$, so that for $A, B \subset \mathbf{R}^{n}$ one has

$$
\delta(A, B):=\inf \{\delta(a, b) \mid(a, b) \in A \times B\} .
$$

The Whitney family $\operatorname{WF}(U)$ of a bounded open set $U \subset \mathbf{R}^{n}$ is given by the subclass of $n$-cubes $K$ of $\mathbf{K}$ such that:

(a) $\delta\left(K, \mathbf{R}^{n} \backslash U\right)>2^{1-\operatorname{level}(K)}$ for each $K \in \mathrm{WF}(U)$;

(b) $\delta\left(L, \mathbf{R}^{n} \backslash U\right) \leq 2^{1-\operatorname{level}(L)}$ for the (unique) element $L$ of $\mathbf{K}$ such that $L$ contains $K$ and level $(L)=\operatorname{level}(K)-1$. 
Therefore, the size of the cubes of $\operatorname{WF}(U)$ is smaller and smaller according to the distance from the boundary of $U$. Moreover one has

(a) $\bigcup \mathrm{WF}(U)=U$;

(b) if $K, L \in \mathrm{WF}(U)$, then $\operatorname{int}(K) \cap \operatorname{int}(L)=\emptyset$;

(c) if $K, L \in \mathrm{WF}(U)$ are such that $\partial K \cap \partial L \neq \emptyset$, then $|\operatorname{level}(K)-\operatorname{level}(L)| \leq 1$;

(d) if $K \in \mathrm{WF}(U)$, then $\partial K \subset \bigcup\{\partial L \mid L \in \mathrm{WF}(U)$ and $L \neq K\}$.

We also recall the notion of cubical complex $\mathrm{CX}(\mathrm{WF}(U))$ associated to the Whitney family $\mathrm{WF}(U)$. For each integer $i=1, \ldots, n$ and for fixed $l \in \mathbf{Z}$, denote by $\mathbf{K}_{i}(l)$ the class of the $i$-dimensional faces of the cubes of $\mathbf{K}(l)$, say $i$-faces of level l. The cubical complex $\mathrm{CX}(\mathrm{WF}(U))$ is given by the faces $F$ of $\bigcup_{i=1}^{n} \bigcup_{l \in \mathbf{Z}} \mathbf{K}_{i}(l)$ for which the following conditions hold:

(a) if $F \in \mathbf{K}_{i}(l)$, then $F$ is an $i$-face of some cube of $\mathrm{WF}(U)$;

(b) if $F \in \mathbf{K}_{i}(l), i \leq n-1$, then $l=\operatorname{level}(F) \geq \operatorname{level}(\widetilde{F})$ for each $i$-face $\widetilde{F}$ of any cube of $\mathrm{WF}(U)$ such that $\operatorname{int}(\widetilde{F}) \cap \operatorname{int}(\bar{F}) \neq \emptyset$.

Define moreover, for each $i \in\{1, \ldots, n\}$,

$$
\mathrm{CX}_{i}(\mathrm{WF}(U)):=\{F \in \mathrm{CX}(\mathrm{WF}(U)) \mid F \text { is of dimension } i\} .
$$

Then, clearly $\mathrm{CX}_{n}(\mathrm{WF}(U))=\mathrm{WF}(U)$ whereas $\mathrm{CX}_{i}(\mathrm{WF}(U))$ selects the $i$-faces which cannot be divided in $2^{i}$ equal $i$-faces of cubes of $\mathrm{WF}(U)$ of smaller size (or higher level). Finally define

$$
\mathrm{CX}_{0}(\mathrm{WF}(U)):=\left\{p \in \mathbf{R}^{n} \mid p \in \partial F \text { and } F \in \mathrm{CX}_{1}(\mathrm{WF}(U))\right\}
$$

i.e., the set of all vertices of the cubes of $\operatorname{WF}(U)$, and define the level of a point $p \in \mathrm{CX}_{0}(\mathrm{WF}(U))$ by the greatest of level $(F)$, as $F$ ranges over the 1-faces of $\mathrm{CX}_{1}(\mathrm{WF}(U))$ for which $p \in \partial F$.

To describe the partition in Proposition 4, we make use of the following

Definition 3. We say that a region $Q$ of $\mathbf{R}^{n}$ is strictly starshaped w.r.t. some interior point $x_{0} \in \operatorname{int}(Q)$ if for each direction $v \in \mathbf{R}^{n},|v|=1$, there is a unique positive $\lambda_{v}>0$ such that the point $x_{0}+\lambda_{v} v$ lies in the boundary $\partial Q$ of $Q, \lambda_{v} \geq \lambda_{0}>0$ and the line segment given by $x_{0}+t \lambda_{v} v, 0 \leq t<1$, lies inside $\operatorname{int}(Q)$.

Proposition 4. There exists a partition of $U$ made by a countable family of polyhedral regions $Q$, pairwise interiorly disjoint, with sides parallel to the coordinate directions and strictly starshaped. Moreover, every polyhedral region belongs to one of the disjoint families $\mathcal{F}_{i}$, for $i \in\{0, \ldots, n\}$, so that for each $i$ there is a 1 : 1 correspondence between elements $\widetilde{Q}$ of $\mathrm{CX}_{i}(\mathrm{WF}(U))$ and polyhedral regions $Q$ of $\mathcal{F}_{i}$ (possibly empty for $i \leq n-1$ ). Also, each region $Q$ is in generic position w.r.t. $u$, see Definition 2 in Sect. 1. More precisely, if $Q \in \mathcal{F}_{i}$ 
and $\widetilde{Q} \in \mathrm{CX}_{i}(\operatorname{WF}(U))$ is the corresponding $i$-face, for each $K \in Q_{(n-j)}$ and $j=1, \ldots, n-2$ the following integral estimate holds

$$
\sum_{K \in Q_{(n-j)}} \int_{K}\left|M\left(D u_{K}\right)\right| d \mathcal{H}^{n-j} \leq c\left(2^{l}\right)^{j} \int_{\Omega_{\tilde{Q}}}|M(D u)| d x,
$$

where $c>0$ is an absolute constant, $l=\operatorname{level}(\widetilde{Q})$ and $\Omega_{\widetilde{Q}}$ is a suitable small neighbor of $\widetilde{Q}$. Finally, the domains $\Omega \widetilde{Q}$ are contained in $U$ and intersect in a finite number, i.e., different $\Omega \widetilde{Q}$ 's, for $\widetilde{Q} \in \mathrm{CX}_{i}(\mathrm{WF}(U))$, are pairwise disjoint if $i=0$ or $i=n$ whereas, if $1 \leq i \leq n-1$, intersect if and only if the corresponding $i$-faces $\widetilde{Q}$ do intersect.

To well understand the construction in Proposition 4, we first consider the case of a partition of all the space $\mathbf{R}^{n}$ w.r.t. a standard grid of $n$-cubes of fixed size $2 \delta>0$

$$
\mathcal{L}=\mathcal{L}(2 \delta):=\bigcup_{z \in \mathbf{Z}^{n}} \delta\left(z+[-1,1]^{n}\right) .
$$

Denote then by $\mathcal{L}_{n-j}$ the $(n-j)$-skeleton of the grid, i.e., the family of $(n-j)$-faces of the $n$-cubes of $\mathcal{L}$, and suppose $u: \mathbf{R}^{n} \rightarrow \mathbf{R}^{N}$ is a map locally in cart ${ }^{1}$, i.e., such that for each bounded domain $\Omega \subset \mathbf{R}^{n}$, the restriction $u_{\mid \Omega}$ is in $\operatorname{cart}^{1}\left(\Omega, \mathbf{R}^{N}\right)$. For each vertex $p \in \mathcal{L}_{0}$, denote by $\Omega_{p}$ the $n$-cube centered at $p$, with sides parallel to the coordinate axes and of length $\delta$

$$
\Omega_{p}:=p+[-\delta / 2, \delta / 2]^{n} .
$$

If $F$ is a $(n-j)$-face of $\mathcal{L}_{n-j}, j \in\{1, \ldots, n-1\}$, denote by $\Omega_{F}$ the $n$ parallelepiped given by the convex envelope of the union of the domains $\Omega_{p}$ corresponding to the vertices $p$ of $F$

$$
\Omega_{F}:=\operatorname{co}\left(\bigcup\left\{\Omega_{p} \mid p \in \mathcal{L}_{0}, \quad p \in \partial F\right\}\right) .
$$

Therefore, if $j \geq 2$ and $F \subset \partial K$, with $K \in \mathcal{L}_{n-j+1}$, we have $\Omega_{F} \subset \Omega_{K}$.

We select, in correspondence to each $(n-1)$-face $K$ of $\mathcal{L}_{n-1}$, an hyperplane $\Pi_{K}$ of $\mathbf{R}^{n}$, parallel to $K$, intersecting the domain $\Omega_{K}$ corresponding to $K, \Pi_{K} \cap \Omega_{K} \neq \emptyset$, so that nice integral estimates hold in $\Pi_{K} \cap \Omega_{K}$ and in the subsets $\Pi_{K} \cap \Omega_{I}$, with $\Omega_{I}$ corresponding to any $(n-j)$-face $I \in \mathcal{L}_{n-j}, j=2, \ldots, n$, which lies in the boundary of $K, I \subset \partial K$ (see (68)). Moreover (since $n \geq 3$ ) the hyperplanes $\Pi_{K}$ 's, for $K \in \mathcal{L}_{n-1}$, are chosen so that if $F \in \mathcal{L}_{n-m-1}$ is any $(n-m-1)$ face, $m=1, \ldots, n-2$, and $\left\{K_{i} \mid i=1, \ldots, m+1\right\}$ is any family of pairwise orthogonal $(n-1)$-faces of $\mathcal{L}_{n-1}$ such that $F=\bigcap_{i=1}^{m+1} K_{i}$, then the $(n-m-1)$ dimensional affine space $\Pi_{F}$ detected by the hyperplanes $\Pi_{K_{i}}$ 's, $\Pi_{F}:=\bigcap_{i=1}^{m+1} \Pi_{K_{i}}$, is such that nice integral estimates hold in $\Pi_{F} \cap \Omega_{F}$ and in the sections $\Pi_{F} \cap \Omega_{I}$, with $\Omega_{I}$ corresponding to any $(n-j)$-face $I \in \mathcal{L}_{n-j}, j=m+2, \ldots, n$, which lies in the boundary of $F, I \subset \partial F$ (see (69)). More precisely, we work in $n$ steps, as follows. 
$1^{\text {st }}$ step: Let $K \in \mathcal{L}_{n-1}$ be any $(n-1)$-face parallel to the hyperplane $\left\{x \in \mathbf{R}^{n} \mid\right.$ $\left.x_{1}=0\right\}$ and $\bar{x}_{1} \in \mathbf{R}$ be such that

$$
K \subset\left\{x \in \mathbf{R}^{n} \mid x_{1}=\bar{x}_{1}\right\} .
$$

Since by Fubini's theorem

$$
\int_{\Omega_{K}}|M(D u)| d x=\int_{\bar{x}_{1}-\delta / 2}^{\bar{x}_{1}+\delta / 2} d \xi \int_{\Omega_{K} \cap\left\{x_{1}=\xi\right\}}|M(D u)| d \mathcal{H}^{n-1},
$$

for $c>0$ large, the measure of the set of points $\xi \in\left(\bar{x}_{1}-\delta / 2, \bar{x}_{1}+\delta / 2\right)$ for which

$$
\int_{\Omega_{K} \cap\left\{x_{1}=\xi\right\}}|M(D u)| d \mathcal{H}^{n-1} \leq \frac{c}{\delta} \int_{\Omega_{K}}|M(D u)| d x
$$

is greater than $(1-1 / c) \delta$. Moreover, if $I \in \mathcal{L}_{n-j}, j=2, \ldots, n$, is any $(n-j)$-face of the boundary of $K, I \subset \partial K$ (hence $\Omega_{I} \subset \Omega_{K}$ ), analogous integral estimates hold with $\Omega_{I}$ instead of $\Omega_{K}$ in (64). Since the number of $(n-j)$-faces of an $(n-1)$-cube is $2^{j-1}\left(\begin{array}{l}n-1 \\ n-j\end{array}\right)$, choosing

$$
c>\sum_{j=1}^{n} 2^{j-1}\left(\begin{array}{l}
n-1 \\
n-j
\end{array}\right)
$$

we are able to select $\xi_{1} \in\left(\bar{x}_{1}-\delta / 2, \bar{x}_{1}+\delta / 2\right)$ so that (64) holds for $\xi=\xi_{1}$ and

$$
\int_{\Omega_{I} \cap\left\{x_{1}=\xi_{1}\right\}}|M(D u)| d \mathcal{H}^{n-1} \leq \frac{c}{\delta} \int_{\Omega_{I}}|M(D u)| d x
$$

for each $I \in \mathcal{L}_{n-j}, I \subset \partial K, j=2, \ldots, n$.

$2^{\text {nd }}$ step: Let now $K \in \mathcal{L}_{n-1}$ be any $(n-1)$-face parallel to the hyperplane $\left\{x \in \mathbf{R}^{n} \mid x_{2}=0\right\}$ and $\bar{x}_{2} \in \mathbf{R}$ be such that

$$
K \subset\left\{x \in \mathbf{R}^{n} \mid x_{2}=\bar{x}_{2}\right\} .
$$

Moreover, let $F_{i} \in \mathcal{L}_{n-2}, i=1,2$, be the $(n-2)$-faces of $K$ parallel to the (n-2)-plane $\left\{x \in \mathbf{R}^{n} \mid x_{1}=x_{2}=0\right\}$ and $\Omega_{F_{i}}$ be the corresponding domains. Also, let $K_{i}^{h} \in \mathcal{L}_{n-1}, h=1,2$, be one of the two $(n-1)$-faces of $\mathcal{L}_{n-1}$ parallel to $\left\{x \in \mathbf{R}^{n} \mid x_{1}=0\right\}$ and such that $K_{i}^{h} \bigcap K=F_{i}$ for each $i, h=1,2$. Finally, let $\xi_{1}(i, h) \in \mathbf{R}$ be chosen in $1^{s t}$ step in correspondence to $K_{i}^{h}$, so that (64) holds with $K=K_{i}^{h}$ and $\xi=\xi_{1}(i, h)$. By Fubini's theorem one has

$$
\begin{aligned}
& \int_{\Omega_{F_{i}} \cap\left\{x_{1}=\xi_{1}(i, h)\right\}}|M(D u)| d \mathcal{H}^{n-1}= \\
& =\int_{\bar{x}_{2}+\delta / 2} d \xi \int_{F_{i} \cap\left\{x_{1}=\xi_{1}(i, h)\right\} \cap\left\{x_{2}=\xi\right\}}|M(D u)| d \mathcal{H}^{n-2},
\end{aligned}
$$


the first member being finite by (65) with $I=F_{i}$ and $\xi_{1}=\xi_{1}(i, h)$, since $F_{i} \subset$ $\partial K_{i}^{h}$. Then, for $c>0$ large, the measure of the set of points $\xi \in\left(\bar{x}_{2}-\delta / 2, \bar{x}_{2}+\delta / 2\right)$ for which

$$
\int_{\Omega_{F_{i}} \cap\left\{x_{1}=\xi_{1}(i, h)\right\} \cap\left\{x_{2}=\xi\right\}}|M(D u)| d \mathcal{H}^{n-2} \leq \frac{c}{\delta} \int_{\Omega_{F_{i}} \cap\left\{x_{1}=\xi_{1}(i, h)\right\}}|M(D u)| d \mathcal{H}^{n-1}
$$

is greater than $(1-1 / c) \delta$, and similarly for $\Omega_{I}$, for each $I \in \mathcal{L}_{n-j}, j=3, \ldots, n$, $I \subset \partial F_{i}$ (hence $\Omega_{I} \subset \Omega_{F_{i}}$ ), with $i, h=1,2$. Since the number of $(n-j)$-faces of an $(n-2)$-cube is $2^{j-2}\left(\begin{array}{c}n-2 \\ n-j\end{array}\right)$, choosing

$$
c>\sum_{j=1}^{n} 2^{j-1}\left(\begin{array}{l}
n-1 \\
n-j
\end{array}\right)+4 \sum_{j=2}^{n} 2^{j-2}\left(\begin{array}{l}
n-2 \\
n-j
\end{array}\right)
$$

we are able to select $\xi_{2} \in\left(\bar{x}_{2}-\delta / 2, \bar{x}_{2}+\delta / 2\right)$ so that on one side

$$
\int_{\Omega_{I} \cap\left\{x_{2}=\xi_{2}\right\}}|M(D u)| d \mathcal{H}^{n-1} \leq \frac{c}{\delta} \int_{\Omega_{I}}|M(D u)| d x
$$

holds for $I=K$ and for each $I \in \mathcal{L}_{n-j}, I \subset \partial K, j=2, \ldots, n$, see $1^{\text {st }}$ step, and on the other side

$$
\int_{\Omega_{I} \cap\left\{x_{1}=\xi_{1}(i, h), x_{2}=\xi_{2}\right\}}|M(D u)| d \mathcal{H}^{n-2} \leq \frac{c}{\delta} \int_{\Omega_{I} \cap\left\{x_{1}=\xi_{1}(i, h)\right\}}|M(D u)| d \mathcal{H}^{n-1}
$$

holds for $I=F_{i}$ and for any $(n-j)$-face $I \in \mathcal{L}_{n-j}, I \subset \partial F_{i}$, with $j=3, \ldots, n$, for each $i, h=1,2$.

Since $n \geq 3$, for each $k=3, \ldots, n$ we have the following

$k^{\text {th }}$ step: Let now $K \in \mathcal{L}_{n-1}$ be any $(n-1)$-face parallel to the hyperplane $\left\{x \in \mathbf{R}^{n} \mid x_{k}=0\right\}$ and $\bar{x}_{k} \in \mathbf{R}$ be such that

$$
K \subset\left\{x \in \mathbf{R}^{n} \mid x_{k}=\bar{x}_{k}\right\} .
$$

Moreover, for fixed $m \in\{1, \ldots, k-1\}(m \leq n-2$ if $k=n)$ and $1 \leq i_{1}<$ $i_{2}<\ldots<i_{m} \leq k-1$, let $F \in \mathcal{L}_{n-m-1}$ be any $(n-m-1)$-face of $K$ parallel to the $(n-m-1)$-plane $\left\{x \in \mathbf{R}^{n} \mid x_{i_{h}}=0 \quad \forall h=1, \ldots, m, x_{k}=0\right\}$. Also, for $h \in\{1, \ldots, m\}$, let $K_{h} \in \mathcal{L}_{n-1}$ be any $(n-1)$-face parallel to the hyperplane $\left\{x \in \mathbf{R}^{n} \mid x_{i_{h}}=0\right\}$ and such that

$$
\bigcap_{h=1}^{m} K_{h} \cap K=F .
$$


If $\xi_{i_{h}} \in \mathbf{R}$ is chosen at $i_{h}^{\text {th }}$ step in correspondence to the $(n-1)$-face $K_{h}$, one has

$$
\begin{gathered}
\int_{\Omega_{F} \cap\left\{x_{i_{h}}=\xi_{i_{h}} \forall h=1, \ldots, m\right\}}|M(D u)| d \mathcal{H}^{n-m}= \\
=\int_{\bar{x}_{k}+\delta / 2} d \xi \quad \int_{\forall / 2}|M(D u)| d \mathcal{H}^{n-m-1} \\
\Omega_{F} \cap\left\{x_{i_{h}}=\xi_{i_{h}} \forall h=1, \ldots, m\right\} \cap\left\{x_{k}=\xi\right\}
\end{gathered}
$$

the first member being finite since by (66) $\Omega_{F} \subset \bigcap_{h=1}^{m} \Omega_{K_{h}}$. Also, a similar integral estimate holds with $\Omega_{I}$ instead of $\Omega_{F}$ in (67), for each $(n-j)$-face $I \in \mathcal{L}_{n-j}$, $j=m+2, \ldots, n, I \subset \partial F$ (hence $\Omega_{I} \subset \Omega_{F}$ ). Since the number of ordered $m$-tuples in $(1, \ldots, k-1)$ is $\left(\begin{array}{c}k-1 \\ m\end{array}\right)$, for $m \in\{1, \ldots, k-1\}$, the $(n-m-1)$-faces $F$ of $K$ parallel to the same $(n-m-1)$-plane are $2^{m}$, the possible choices of distinct $(n-1)$-faces $K_{h}$ parallel to $\left\{x_{i_{h}}=0\right\}$ for which (66) holds are $2^{m}$, for each $h \in\{1, \ldots, m\}$, and finally the number of $(n-j)$-faces $I$ of the $(n-m-1)$-face $F$, for $j=m+2, \ldots, n$, is $2^{j-m-1}\left(\begin{array}{c}n-m-1 \\ n-j\end{array}\right)$, if we choose

$$
c>\sum_{j=1}^{n} 2^{j-1}\left(\begin{array}{c}
n-1 \\
n-j
\end{array}\right)+\sum_{m=1}^{k-1}\left(\begin{array}{c}
k-1 \\
m
\end{array}\right) m 2^{2 m} \sum_{j=m+1}^{n} 2^{j-m-1}\left(\begin{array}{c}
n-m-1 \\
n-j
\end{array}\right)
$$

we are then able to select $\xi_{k} \in\left(\bar{x}_{k}-\delta / 2, \bar{x}_{k}+\delta / 2\right)$ so that, on one side,

$$
\int_{\Omega_{I} \cap\left\{x_{k}=\xi_{k}\right\}}|M(D u)| d \mathcal{H}^{n-1} \leq \frac{c}{\delta} \int_{\Omega_{I}}|M(D u)| d x
$$

holds for $I=K$ and for each $I \in \mathcal{L}_{n-j}, I \subset \partial K, j=2, \ldots, n$, as in $1^{s t}$ step, and on the other side for each $m \in\{1, \ldots, k-1\}(m \leq n-2)$, for any choice of $1 \leq i_{1}<i_{2}<\ldots<i_{m} \leq k-1$, for each $(n-m-1)$-face $F \in \mathcal{L}_{n-m-1}$ of $K$ parallel to $\left\{x_{i_{h}}=0 \quad \forall h=1, \ldots, m, x_{k}=0\right\}$, for any choice of $K_{h} \in \mathcal{L}_{n-1}$ parallel to $\left\{x_{i_{h}}=0\right\}$ and for which (66) holds, then

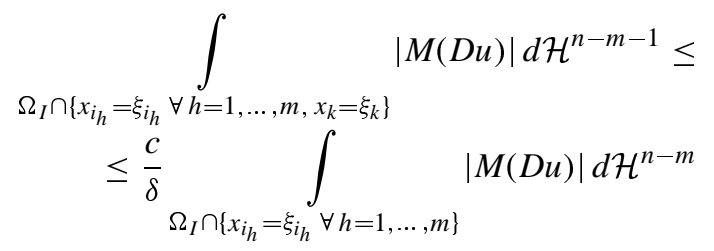

holds for $I=F$ and for each $I \in \mathcal{L}_{n-j}, I \subset \partial F, j=m+2, \ldots, n$, where $\xi_{i_{h}} \in \mathbf{R}$ is chosen at $i_{h}{ }^{\text {th }}$ step in correspondence to the $(n-1)$-face $K_{h}$.

To prove Proposition 4, we make an adapation of the previous construction to the case of a Whitney family $\mathrm{WF}(U)$ associated to a bounded open set $U \subset \mathbf{R}^{n}$, and obtain analogous integral estimates. Here, the role of the $(n-j)$-skeleton $\mathcal{L}_{n-j}$ is played by the $(n-j)$-dimensional cubical complex $\mathrm{CX}_{n-j}(\mathrm{WF}(U))$. 
Proof of Proposition 4. For each vertex $p \in \mathrm{CX}_{0}(\mathrm{WF}(U))$, denote by $\Omega_{p}$ the $n$ cube centered at $p$, with sides parallel to the coordinate axes and of length $2^{-(l+1)}$, where $l=\operatorname{level}(p)$

$$
\Omega_{p}:=p+\left[-2^{-(l+2)}, 2^{-(l+2)}\right]^{n} .
$$

Let $F \in \mathrm{CX}_{n-j}(\mathrm{WF}(U))$ be any $(n-j)$-face of the cubical complex, $j=$ $1, \ldots, n-1$, with level $(F)=l$, and let

$$
\left\{p_{i} \mid i=1, \ldots, 2^{n-j}\right\} \subset \mathrm{CX}_{0}(\mathrm{WF}(U))
$$

denote the set of vertices of $F$. We distinguish two cases.

If all the vertices $p_{i}$ of $F$ have the same level, denote by $\Omega_{F}$ the $n$-parallelepiped given by the convex envelope of the union of the $\Omega_{p_{i}}$ 's

$$
\Omega_{F}:=\operatorname{co}\left(\bigcup_{i=1}^{2^{n-j}} \Omega_{p_{i}}\right) .
$$

If not all the vertices $p_{i}$ of $F$ have the same level, i.e., level $\left(p_{i}\right)$ is equal to $l$ for some $p_{i}$ 's and to $l+1$ for other ones, we denote by $\widetilde{\Omega}_{p_{i}}$, for each $i=1, \ldots, 2^{n-j}$, the $n$-parallelepiped centered at $p_{i}$, with sides parallel to the sides of $F$ of length $2^{-(l+1)}$ and sides orthogonal to $F$ of half length $2^{-(l+2)}$ (thus $\widetilde{\Omega}_{p_{i}}$ depends on $F$ ) and define $\Omega_{F}$ as in (70), with $\widetilde{\Omega}_{p_{i}}$ instead of $\Omega_{p_{i}}$.

The integral estimates are obtained similarly to the case of the standard grid of mesh $2 \delta$, taking account that for each $K \in \mathrm{CX}_{n-1}(\mathrm{WF}(U))$, the size $\delta$ is equal to $2^{-(l+1)}$ or $2^{-(l+2)}, l=\operatorname{level}(K)$, according to the length of the sides of $\Omega_{K}$ orthogonal to $K$. Moreover, note that the combinatorial complexity of $\mathrm{CX}(\operatorname{WF}(U))$ is dominated by the case of a standard grid $\mathcal{L}$. We then reach the following conclusions.

For each $k=1, \ldots, n$, at $k^{\text {th }}$ step, for any $(n-1)$-face $K$ of the cubical complex $\mathrm{CX}_{n-1}(\mathrm{WF}(U))$ parallel to $\left\{x \mid x_{k}=0\right\}$, say $K \subset\left\{x \mid x_{k}=\bar{x}_{k}\right\}$, we can select $\xi_{k} \in\left(\bar{x}_{k}-2^{-(l+2)}, \bar{x}_{k}+2^{-(l+2)}\right)$ (or $2^{-(l+3)}$ if the side of $\Omega_{K}$ orthogonal to $K$ is of length $2^{-(l+2)}$ ), where $l=\operatorname{level}(K)$, such that (68) holds, with $\delta=2^{-l}$, for $I=K$ and for each $I \in \mathrm{CX}_{n-j}(\mathrm{WF}(U)), j=2, \ldots, n$, $I \subset \partial K$, where $c>0$ is an absolute constant. Moreover, in case $k>1$, for each $m \in\{1, \ldots, k-1\}(m \leq n-2$ if $k=n)$, for any choice of $1 \leq i_{1}<i_{2}<\ldots<$ $i_{m} \leq k-1$, let $F \in \mathrm{CX}_{n-m-1}(\mathrm{WF}(U))$ be any $(n-m-1)$-face contained in $\partial K$ and parallel to $\left\{x \mid x_{i_{h}}=0 \quad \forall h=1, \ldots, m, x_{k}=0\right\}$. Then, for any choice of $K_{h} \in \mathrm{CX}_{n-1}(\mathrm{WF}(U))$, parallel to $\left\{x_{i_{h}}=0\right\}$ and for which (66) holds, we infer that (69) holds, with $\delta=2^{-l}$, for $I=F$ and for each $I \in \mathrm{CX}_{n-j}(\mathrm{WF}(U))$, $I \subset \partial F, j=m+2, \ldots, n$, where $\xi_{i_{h}} \in \mathbf{R}$ is chosen at $i_{h}{ }^{\text {th }}$ step in correspondence to the $(n-1)$-face $K_{h}$.

Remark 7. Actually, since $n \geq 3$, if $k \geq n-1$ and $m=n-2$, for each $F \in \mathrm{CX}_{1}(\mathrm{WF}(U))$ as before, it suffices to require finiteness of the 1-dimensional integrals in the left-hand side of (69). 
By means of suitable intersections of the previously defined $(n-1)$-faces $\Omega_{K} \cap\left\{x_{k}=\xi_{k}\right\}, k \in\{1, \ldots, n\}, K \in \mathrm{CX}_{n-1}(\mathrm{WF}(U))$, it is possible to define the partition of the open subset $U \subset \Omega$ as described in Proposition 4. In particular, one infers that (63) follows by (68) and by iterated applications of (69), where $\delta=2^{-l}$.

Remark 8. For future purposes, we finally note that the polyhedral regions $Q$ of the partition in Proposition 4 have the following size upper bounds. If $Q \in \mathcal{F}_{i}$ and $\widetilde{Q} \in \mathrm{CX}_{i}(\mathrm{WF}(U))$ is the corresponding $i$-face, with level $(\widetilde{Q})=l$, then the diameter of $Q$ is smaller than $\sqrt{n} 2^{-l+1}$. Moreover, $Q$ is strictly starshaped w.r.t. some point $x_{0} \in \operatorname{int}(Q)$ so that, if $\lambda_{v}>0$ corresponds to $v \in \mathbf{R}^{n},|v|=1$, see Definition 3, then one has

$$
\sup \left\{\lambda_{v}\left|v \in \mathbf{R}^{n},\right| v \mid=1\right\} \leq c 2^{-l}
$$

for some absolute constant $c>0$.

\section{Proof of the main result}

To show the "if" part, we recall by [13] that every continuous map in $\operatorname{cart}^{1}\left(\Omega, \mathbf{R}^{N}\right)$ can be approximated by smooth maps. We therefore reduce to prove that if $u \in$ $\operatorname{cart}^{1}\left(\Omega, \mathbf{R}^{N}\right)$ is such that the restriction of $u$ to a.e. 2-plane can be approximated by smooth maps, then we can define, for each small $\varepsilon>0$, a continuous map $u_{\varepsilon}: \Omega \rightarrow \mathbf{R}^{N}$ in $\operatorname{cart}^{1}\left(\Omega, \mathbf{R}^{N}\right)$ such that $u_{\varepsilon}$ is equal to $u$ in $\Omega \backslash U_{\varepsilon}$, with $U_{\varepsilon}$ bounded open set of arbitrary small measure, and such that, by absolute continuity of the area integral (1)

$$
\mathrm{A}\left(u_{\varepsilon}, U_{\varepsilon}\right) \leq \mathrm{A}\left(u, U_{\varepsilon}\right)+c \varepsilon
$$

where $c>0$ is an absolute constant.

Recall to this aim that by Liu's theorem [10], if $u \in \operatorname{cart}^{1}\left(\Omega, \mathbf{R}^{N}\right)$, for each small $\varepsilon>0$ there is a closed set $C_{\varepsilon} \subset \Omega$ such that $\left|\Omega \backslash C_{\varepsilon}\right| \leq \varepsilon$ and $u$ is of class $C^{1}$ in $C_{\varepsilon}$. We then consider the Whitney family $\operatorname{WF}\left(U_{\varepsilon}\right)$ of cubes associated to the open set

$$
U_{\varepsilon}:=\Omega \backslash C_{\varepsilon}
$$

and partition the singular set $U_{\varepsilon}$ in a countable family of polyhedral regions as in Proposition 4 of Sect. 4.

Consequently, if $Q \in \mathcal{F}_{i}$ is any region of the partition, with $i \in\{0, \ldots, n\}$, and $\widetilde{Q} \in \mathrm{CX}_{i}\left(\mathrm{WF}\left(U_{\varepsilon}\right)\right)$ corresponds to $Q$, then (63) holds for each $(n-j)$-face $K$ of $Q_{(n-j)}$, with $j=1, \ldots, n-2$. Moreover, by the generic position of $Q$ w.r.t. $u$, see Definition 2 in Sect. 1, by an obvious generalization of Proposition 2 in Sect. 2, we infer that conditions i) and ii) at the beginning of Sect. 3, in particular (22), (23) and (24), hold for the restriction $u_{F}:=u_{\mid F}$ of $u$ to any 2-face $F$ of $Q_{(2)}$.

Then, by an adaptation of the local approximation argument in Sect. 3, Proposition 3 holds for any polyhedral region $Q$ of the partition of $U_{\varepsilon}$. More precisely, if $Q$ is strictly starshaped w.r.t. $x_{0} \in \operatorname{int}(Q)$, see Definition 3 in Sect. 4, up to a translation we set $x_{0}=0_{\mathbf{R}^{n}}$. For each $k=3, \ldots, n$, if $K \in Q_{(k-1)}$ is a $(k-1)$-face of $Q$, 
for example $K=\widetilde{K} \times\{\widehat{\delta}\}$, where $\widetilde{K}$ is a $(k-1)$-dimensional polyhedral region of $\mathbf{R}^{k-1}$ and $\widehat{\delta} \in \mathbf{R}^{n-k+1}$ is fixed, we modify $k^{\text {th }}$ step of the proof of Proposition 3 by setting

$$
\|\widetilde{x}\|_{k-1}:=\inf \left\{\alpha>0 \mid \frac{\delta}{\alpha} \tilde{x} \in \widetilde{K}\right\}, \quad \tilde{x} \in \mathbf{R}^{k-1},
$$

thus $(\widetilde{x}, \widehat{\delta}) \in K$ if and only if $\|\widetilde{x}\|_{k-1} \leq \delta$,

$$
\Delta_{K}^{1}:=\left\{\left(\tilde{x}, \frac{t}{\delta} \widehat{\delta}\right) \mid \delta\left(\frac{\lambda+1}{2}\right)<t \leq \delta, \quad\|\widetilde{x}\|_{k-1} \leq \delta f(t)\right\}
$$

and

$$
\begin{aligned}
& \left.\left.G^{(1)}:\right] \delta\left(\frac{\lambda+1}{2}\right), \delta\right] \times \widetilde{K} \longrightarrow \Delta_{K}^{1} \times \mathbf{R}^{N} \\
& G^{(1)}(t, \widetilde{x}):=\left(\left(f(t) \widetilde{x}, \frac{t}{\delta} \widehat{\delta}\right), v_{K}(\widetilde{x}, \widehat{\delta})\right)
\end{aligned}
$$

for example, where $\delta=2^{-l}, l=\operatorname{level}(\widetilde{Q})$, with $\widetilde{Q} \in \mathrm{CX}_{i}\left(\operatorname{WF}\left(U_{\varepsilon}\right)\right)$ corresponding to $Q \in \mathcal{F}_{i}$ as in Proposition 4. Moreover, Proposition 1 easily extends to any $k$-face $L$ of $Q_{(k)}$, with $k \geq 3$, whereas, taking account of Remark 8 at the end of Sect. 4, (46) and (62) hold again (with $j=1, \ldots, k, L$ for $Q,\|\cdot\|_{k}$ given as in (71) w.r.t. $L$ ) and so on.

Therefore, for each region $Q$, there exists a map $v_{Q}: Q \rightarrow \mathbf{R}^{N}$ in $\operatorname{cart}^{1}\left(Q, \mathbf{R}^{N}\right)$, continuous up to the closure of $Q$, such that the restriction of $v_{Q}$ to each $(n-j)$-face $K$ of $Q_{(n-j)}, j=1, \ldots, n-1$, is a map in $\operatorname{cart}^{1}\left(K, \mathbf{R}^{N}\right)$ which only depends on the restriction $u_{K}$ of $u$ to $K$ and finally

$$
\begin{aligned}
\mathbf{M}\left(G_{v_{Q}}\left\llcorner Q \times \mathbf{R}^{N}\right)\right. & \leq \mathbf{M}\left(G_{u}\left\llcorner Q \times \mathbf{R}^{N}\right)+\sigma\right. \\
& +\widetilde{c} \sum_{j=1}^{n-2}\left(2^{-l}\right)^{j} \sum_{K \in Q_{(n-j)}} \int_{K}\left|M\left(D u_{K}\right)\right| d \mathcal{H}^{n-j}
\end{aligned}
$$

where $\tilde{c}>0$ is an absolute constant, $\sigma>0$ is chosen small and $l=\operatorname{level}(\widetilde{Q})$, with $\widetilde{Q} \in \mathrm{CX}_{i}\left(\mathrm{WF}\left(U_{\varepsilon}\right)\right)$ corresponding to the region $Q \in \mathcal{F}_{i}$.

Let now $m_{l}, l \in \mathbf{Z}$, denote the number of $n$-cubes of level $l$ of $\operatorname{WF}\left(U_{\varepsilon}\right)$. Since $\left|U_{\varepsilon}\right|<\varepsilon$, clearly $m_{l}=0$ if $l<l_{0}:=\left[\log _{2}\left(\varepsilon^{-1 / n}\right)\right]$ whereas, for $l \geq l_{0}$, $m_{l} \leq \varepsilon\left(2^{l}\right)^{n}$. Moreover, since each $n$-cube has $2^{n-i}\left(\begin{array}{l}n \\ i\end{array}\right) i$-faces, the number of $i$-faces of level $l$ of $\mathrm{CX}_{i}(\mathrm{WF}(U))$ is at most $2^{n-i}\left(\begin{array}{l}n \\ i\end{array}\right) m_{l}$. Then, by summation w.r.t. $Q \in \mathcal{F}_{i}$, taking $\sigma=\left[2^{n-i}\left(\begin{array}{l}n \\ i\end{array}\right) m_{l}\right]^{-1} \varepsilon$ in (72) if $Q \in \mathcal{F}_{i}$ and $l=\operatorname{level}(\widetilde{Q})$, with $\widetilde{Q} \in \mathrm{CX}_{i}\left(\mathrm{WF}\left(U_{\varepsilon}\right)\right)$ corresponding to $Q$, by (72) and (63) one infers, for each $i \in\{0, \ldots, n\}$,

$$
\begin{aligned}
\sum_{Q \in \mathcal{F}_{i}} \mathbf{M}\left(G_{v_{Q}}\left\llcorner Q \times \mathbf{R}^{N}\right)\right. & \leq \sum_{Q \in \mathcal{F}_{i}} \mathbf{M}\left(G_{u}\left\llcorner Q \times \mathbf{R}^{N}\right)+\varepsilon\right. \\
& +c \tilde{c}(n-2) \sum_{Q \in \mathcal{F}_{i}} \int_{\Omega_{\widetilde{Q}}}|M(D u)| d x .
\end{aligned}
$$

Finally, for fixed $i$, since the domains $\Omega \widetilde{Q}$, for $\widetilde{Q} \in \mathrm{CX}_{i}\left(\mathrm{WF}\left(U_{\varepsilon}\right)\right)$, are contained in $U_{\varepsilon}$ and are pairwise disjoint if $i=0$ or $i=n$ whereas, if $1 \leq i \leq n-1$, 
different $\Omega \widetilde{Q}$ 's intersect if and only if the corresponding $i$-faces $\widetilde{Q}$ do intersect (see Proposition 4), one has

$$
\sum_{Q \in \mathcal{F}_{i}} \mathbf{M}\left(G_{v_{Q}}\left\llcorner Q \times \mathbf{R}^{N}\right) \leq[1+c \tilde{c}(n-2) \widehat{c}] \mathbf{M}\left(G_{u}\left\llcorner U_{\varepsilon} \times \mathbf{R}^{N}\right)+\varepsilon\right.\right.
$$

where $\widehat{c}=\widehat{c}(n, i)$ is an absolute constant. Then, taking $u_{\varepsilon} \equiv u$ in $\Omega \backslash U_{\varepsilon}$ and $u_{\varepsilon}=v_{Q}$ in $Q$, for each domain $Q \in \mathcal{F}_{i}, i \in\{0, \ldots, n\}$, the mapping $u_{\varepsilon}: \Omega \rightarrow \mathbf{R}^{N}$, for $\left\{\varepsilon_{j}\right\} \downarrow 0$, defines the approximating sequence.

In fact, by summation on $i \in\{0, \ldots, n\}$ in (73) one infers

$$
\mathbf{M}\left(G_{u_{\varepsilon}}\left\llcorner U_{\varepsilon} \times \mathbf{R}^{N}\right) \leq C \mathbf{M}\left(G_{u}\left\llcorner U_{\varepsilon} \times \mathbf{R}^{N}\right)+(n+1) \varepsilon\right.\right.
$$

where $C:=(n+1)+c \tilde{c}(n-2) \sum_{i=0}^{n} \widehat{c}(n, i)$ is an absolute constant. Also, since $u_{\varepsilon}$ is continuous in $Q$ up to the closure, for each region $Q$ of the partition of $U_{\varepsilon}$, with $u_{\varepsilon \mid K}$ depending on $u_{\mid K}$, for each $(n-j)$-face $K$ of $Q_{(n-j)}, j=1, \ldots, n$, then $u_{\varepsilon}$ is continuous on $U_{\varepsilon}$. Moreover, $u$ is Lipschitz-continuous in $\Omega \backslash U_{\varepsilon}$ and $u_{\varepsilon}$ coincides with $u$ on the 1 -skeleton of each region $Q$, see Proposition 3 in Sect. 3, with $Q$ smaller and smaller according to its distance from $\Omega \backslash U_{\varepsilon}$, compare Remark 8 at the end of Sect. 4 . We then conclude that $u_{\varepsilon}$ is continuous in $\Omega$. Also, $u_{\varepsilon \mid Q} \in \operatorname{cart}^{1}\left(Q, \mathbf{R}^{N}\right)$ for each region $Q$ whereas, if $F$ is a common $(n-1)$-face between two regions $Q_{1}, Q_{2}$, by Proposition 3 we have

$$
\partial\left(G _ { u _ { \varepsilon } } \llcorner Q _ { 1 } \times \mathbf { R } ^ { N } ) \left\llcornerF \times \mathbf{R}^{N}=-\partial\left(G _ { u _ { \varepsilon } } \llcorner Q _ { 2 } \times \mathbf { R } ^ { N } ) \left\llcornerF \times \mathbf{R}^{N} .\right.\right.\right.\right.
$$

Therefore, since there is no "creation" of boundary when defining $u_{\varepsilon}$ on $U_{\varepsilon}$, whereas $u_{\varepsilon}=u$ is Lipschitz in the closed set $\Omega \backslash U_{\varepsilon}$, we thus infer

$$
\partial G_{u_{\varepsilon}}\left\llcorner\Omega \times \mathbf{R}^{N}=0\right.
$$

and then $u_{\varepsilon} \in \operatorname{cart}^{1}\left(\Omega, \mathbf{R}^{N}\right)$. Finally, since $\left|U_{\varepsilon}\right|<\varepsilon$, by absolute continuity of the area integral (1) and by (74), one infers that $\left\{u_{\varepsilon}\right\}$ converges strongly in $L^{1}$ with all minors to $u$, as $\varepsilon \rightarrow 0^{+}$. Since by [13] each continuous Cartesian map $u_{\varepsilon}$ can be approximated by smooth maps, the claim is proved.

Acknowledgements. I wish to thank M. Giaquinta and G. Modica for useful discussions and suggestions.

\section{References}

1. Acerbi, E., Dal Maso, G.: New lower semicontinuity results for polyconvex integrals. Calc. Var. 2, 329-372 (1994)

2. Almgren, F.J.: Deformations and multi-valued functions. Proc. Sympos. Pure Math. 44 A.M.S., Providence, R.I. 29-130 (1986)

3. Federer, H.: Geometric measure theory. Grundlehren Math. Wissen. 153, Berlin: Springer 1969

4. Giaquinta, M., Modica, G., Souček, J.: Cartesian currents, weak diffeomorphisms and existence theorems in nonlinear elasticity. Arch. Rat. Mech. Anal. 106, 97-159 (1989) Erratum and addendum. Arch. Rat. Mech. Anal. 109, 385-392 (1990) 
5. Giaquinta, M., Modica, G., Souček, J.: Cartesian currents and variational problems for mappings into spheres. Ann. Sc. Norm. Sup. Pisa 16, 393-485 (1989)

6. Giaquinta, M., Modica, G., Souček, J.: Graphs of finite mass which cannot be approximated in area by smooth graphs. Manuscr. Math. 78, 259-271 (1993)

7. Giaquinta, M., Modica, G., Souček, J.: Area and area formulas. Rend. Sem. Mat. Milano 42, 53-87 (1992)

8. Giaquinta, M., Modica, G., Souček, J.: Cartesian currents in the calculus of variations, I, II. Ergebnisse Math. Grenzgebiete (III Ser). Berlin: Springer 1998

9. Hu, S.T.: Homotopy theory. New York: Academic Press 1959

10. Liu, F.: A Lusin type property of Sobolev functions. Indiana Univ. Math. J. 26, 645-651 (1977)

11. Malý, J.: $L^{p}$-approximation of Jacobians. Comment. Math. Univ. Carolinae 32(4), 659-666 (1991)

12. Mucci, D.: Approximation in area of graphs with isolated singularities. Manuscr. Math. 88, 135-146 (1995)

13. Mucci, D.: Approximation in area of continuous graphs. Calc. Var. 4, 525-557 (1996)

14. Mucci, D.: Graphs of finite mass which cannot be approximated by smooth graphs with equibounded area. J. Func. Anal. 152, 467-480 (1998)

15. Reshetnyak, Y.G.: Weak convergence of completely additive vector functions on a set. Sib. Math. J. 9, 1039-1045 (1968)

16. Simon, L.: Lectures on geometric measure theory. Proc. C.M.A. 3, Australian Natl. U. (1983)

17. White, B.: Existence of least-area mappings of N-dimensional domains. Ann. Math. (2) 118, 179-185 (1983) 